\title{
Optical conductivity of black phosphorus with a tunable electronic structure
}

\author{
Jiho Jang, ${ }^{1}$ Seongjin Ahn, ${ }^{1,2}$ and Hongki Min ${ }^{1}$ \\ ${ }^{1}$ Department of Physics and Astronomy, Seoul National University, Seoul 08826, Korea \\ ${ }^{2}$ Center for Correlated Electron Systems, Institute for Basic Science (IBS), Seoul 08826, Korea
}

(Dated: March 11, 2019)

\begin{abstract}
Black phosphorus (BP) is a two-dimensional layered material composed of phosphorus atoms. Recently, it was demonstrated that external perturbations such as an electric field close the band gap in few-layer BP, and can even induce a band inversion, resulting in an insulator phase with a finite energy gap or a Dirac semimetal phase characterized by two separate Dirac nodes. At the transition between the two phases, a semi-Dirac state appears in which energy disperses linearly along one direction and quadratically along the other. In this work, we study the optical conductivity of few-layer BP using a lattice model and the corresponding continuum model, incorporating the effects of an external electric field and finite temperature. We find that the low-frequency optical conductivity scales a power law that differs depending on the phase, which can be utilized as an experimental signature of few-layer BP in different phases. We also systematically analyze the evolution of the material parameters as the electric field increases, and the consequence on the power-law behavior of the optical conductivity.
\end{abstract}

\section{MOTIVATION}

Black phosphorus (BP) is a two-dimensional (2D) layered material composed of phosphorus atoms, where the layers are stabilized by weak van der Waals forces, and thus can be exfoliated into a few-layer form. (For a recent review, see 11.) It is known that the band gap of $\mathrm{BP}$ decreases as the thickness increases from $1.6 \mathrm{eV}$ for a monolayer to $0.3 \mathrm{eV}$ in bulk [2 4]. Recently, it was discovered that external perturbations such as pressure [5, 6], strain [7, an electric field [8 10] and surface doping [11-13] close the band gap, and can even induce band inversion in few-layer BP. This results in three different states in few-layer BP: an insulator phase with a gap, a semi-Dirac point with gapless anisotropic dispersion (linear and quadratic in the armchair and zigzag directions, respectively), and a Dirac semimetal phase with two Dirac points, as illustrated in Fig. 1

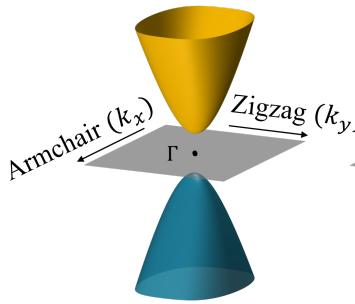

(a)

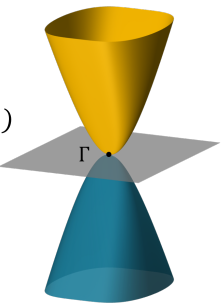

(b)

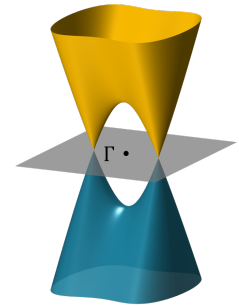

(c)
FIG. 1: Low-energy band structure of few-layer BP in the (a) insulator phase, (b) semi-Dirac point, and (c) Dirac semimetal phase.

There have been a significant number of theoretical and experimental studies concerning various physical properties of few-layer BP, including the electronic structure 14 16], optical properties [17 25], transport properties [19, 26, 29], and Landau levels [9, 30-33. The tightbinding model for few-layer BP has been proposed by several groups [34 36].

However, to our knowledge there has been no systematic study on the optical conductivity of few-layer BP in each phase and the corresponding characteristic frequency dependence. In this study, we investigate the optical conductivity of few-layer $\mathrm{BP}$ with $\mathrm{AB}$ stacking type, which is the most common and energetically stable stacking configuration [37, 38. We conduct both numerical and analytical calculations using a lattice model and the corresponding continuum model, which contain the two phases and capture all the low-energy optical properties. As the perpendicular external electric field increases, the self-consistently obtained energy gap parameter $\varepsilon_{\mathrm{g}}$ changes from a positive to a negative value, and the optical conductivity exhibits a characteristic frequency dependence in each phase for both armchair and zigzag directions, which can be utilized optically to identify each phase in few-layer BP.

This paper is organized as follows. In Sec. II] we introduce the tight-binding lattice model Hamiltonian and continuum model Hamiltonian for few-layer BP. In Sec. III] we study the influence of an external electric field on few-layer BP within a mean-field Hartree approximation, and self-consistently obtain the electronic band structure, as well as the evolution of the model parameters with the external electric field. In Sec. IV] we present the results calculated for the optical conductivity based on the two models along with the analytic results for each phase. We also describe the effect of a finite temperature on the optical conductivity, demonstrating a power-law change at low frequencies. Finally, in Sec V we conclude with a discussion on the effect of the number of layers and the intraband response. 


\section{MODEL}

\section{A. Lattice model}

In this section, we introduce a tight-binding model for few-layer BP, along with its crystal structure shown in Fig. 2. Few-layer BP exhibits a buckled honeycomb lattice structure, with four phosphorus atoms in each unit cell. The tight-binding lattice model for few-layer BP in the basis of sublattices with $3 s, 3 p_{x}$, and $3 p_{z}$ orbitals is given by

$$
H=\sum_{l, i} \varepsilon_{l, i} c_{l, i}^{\dagger} c_{l, i}+\sum_{l, i \neq j} t_{i, j}^{l} c_{l, i}^{\dagger} c_{l, j}+\sum_{l \neq m, i, j} t_{i, j}^{l, m} c_{l, i}^{\dagger} c_{m, j}(1)
$$

where $c_{l, i}^{\dagger}\left(c_{l, i}\right)$ corresponds to the creation (annihilation) operator for an electron on the $i$ th site in the $l$ th layer, and $t_{i, j}^{l}$ and $t_{i, j}^{l, m}$ are intralayer and interlayer hopping parameters, respectively. Here, $\varepsilon_{l, i}$ is the on-site energy, which is self-consistently determined in the presence of an external perpendicular electric field, as will be discussed in Sec. III. In this work, we consider intralayer hopping terms for up to 10 nearest neighbors, and the five nearestneighbor interlayer hopping terms given in [35].

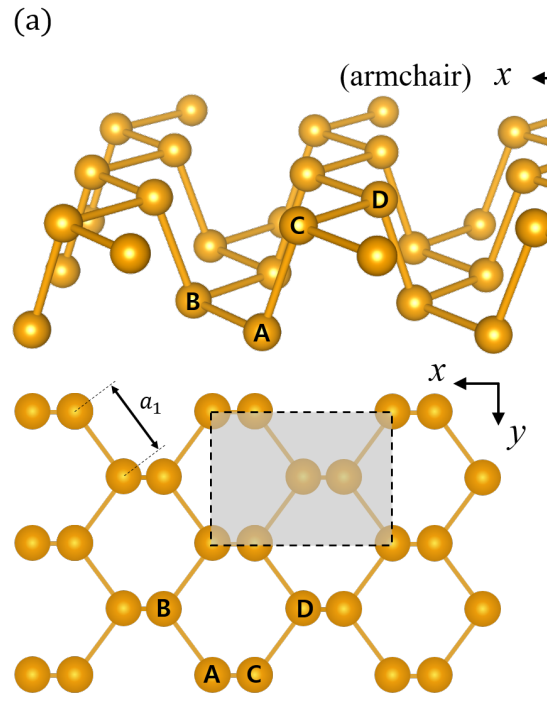

(b)

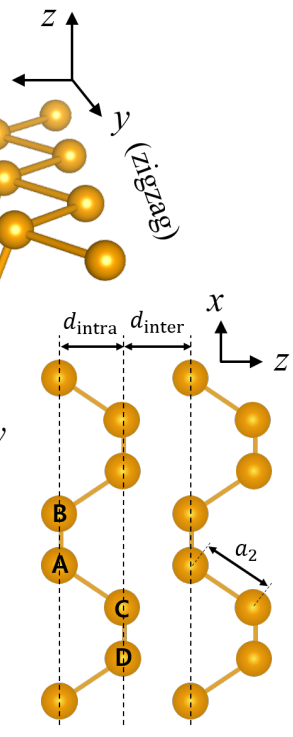

(c)
FIG. 2: (a) Atomic structure of monolayer BP and (b) its top view. (c) Side view of bilayer BP. Here, the $x$ and $y$ axes are set along the armchair and zigzag directions, respectively. The shaded rectangle in (b) indicates the unit cell of BP. The interatomic distances $a_{1}$ and $a_{2}$ are given by $a_{1}=2.21 \AA$ and $a_{2}=2.24 \AA$, and the interlayer and intralayer distances are given by $d_{\text {inter }}=3.17 \AA$ and $d_{\text {intra }}=2.13 \AA$, respectively.

\section{B. Continuum model}

The low-energy expansion of the tight-binding Hamiltonian in Eq. (1) around the $\Gamma$ point yields

$$
H=\left(\begin{array}{cc}
\varepsilon_{\mathrm{CB}}+a_{x} k_{x}^{2}+a_{y} k_{y}^{2} & -i t k_{x} \\
i t^{*} k_{x} & \varepsilon_{\mathrm{VB}}+b_{x} k_{x}^{2}+b_{y} k_{y}^{2}
\end{array}\right),
$$

where $\varepsilon_{\mathrm{CB}}$ is the conduction band minimum and $\varepsilon_{\mathrm{VB}}$ is the valence band maximum. Note that linear terms in $k_{y}$ are not allowed in the off-diagonal element of the Hamiltonian, owing to the reflection symmetry with respect to the $y=0$ plane $\left(\mathcal{M}_{y}\right)$ [7, 13]. From the energy dispersion of the tight-binding model, we confirm that we can effectively set $a_{x} \approx-b_{x}$ and $a_{y} \approx-b_{y}$ [12, leading to

$$
H=\hbar v k_{x} \sigma_{y}+\left(\frac{1}{2} \varepsilon_{\mathrm{g}}+\gamma \frac{\hbar^{2} k_{x}^{2}}{2 m}+\frac{\hbar^{2} k_{y}^{2}}{2 m}\right) \sigma_{z}
$$

where $\varepsilon_{\mathrm{g}}=\varepsilon_{\mathrm{CB}}-\varepsilon_{\mathrm{VB}}, v$ is the effective velocity along the armchair direction, and $m$ is the effective mass along the zigzag direction. Here, we set $\frac{1}{2}\left(\varepsilon_{\mathrm{CB}}+\varepsilon_{\mathrm{VB}}\right)=0$ to be the zero of the energy.

Note that the parabolic term $\gamma \frac{\hbar^{2} k_{x}^{2}}{2 m} \sigma_{z}$, whose contribution is characterized by the dimensionless parameter $\gamma$, is added in the low-energy continuum model in the armchair direction beyond the lowest-order linear term $\hbar v k_{x} \sigma_{y}$. We included this term to take into account its role in the optical conductivity, especially at high frequencies, as will be discussed in Sec. IV.

Figure 1 illustrates the energy dispersions depending on the $\operatorname{sign}$ of $\varepsilon_{\mathrm{g}}$. When $\varepsilon_{\mathrm{g}}>0$, the system is in the insulator phase, and $\varepsilon_{\mathrm{g}}$ corresponds to the size of the energy gap [Fig. 11(a)]. When the band gap closes $\left(\varepsilon_{\mathrm{g}}=0\right)$, the system is described by a 2D semi-Dirac Hamiltonian [39, 40] [Fig. 1 (b)], where the energy dispersion is linear along the armchair direction $\left(k_{x}\right)$ and quadratic along the zigzag direction $\left(k_{y}\right)$. When $\varepsilon_{\mathrm{g}}<0$, a band inversion occurs, and the semi-Dirac point splits into two separated Dirac points located at $\boldsymbol{k}=\left(0, \pm \sqrt{\frac{m\left|\varepsilon_{\mathrm{g}}\right|}{\hbar^{2}}}\right)$ [Fig. 11(c)].

\section{SCREENING THEORY}

In this section, we explore the relation between the band structure of few-layer BP and its dual-gate configuration within a self-consistent Hartree approximation. We consider the situation in which few-layer BP is located between the two metallic gates, whose charge densities are given by $n_{\mathrm{tg}} \leq 0$ (top gate) and $n_{\mathrm{bg}} \geq 0$ (bottom gate). By tuning the gate voltages, one can manipulate both the electric field applied to the few-layer BP and the gate-induced charge density in each layer of BP. In the following, we explain the self-consistent Hartree formalism for few-layer BP, and present numerical results obtained by solving the self-consistent Hartree equation. 


\section{A. Self-consistent Hartree approximation}

We begin with the non-interacting Hamiltonian for a layered system,

$$
H_{0}=\sum_{\boldsymbol{k}, \lambda, \lambda^{\prime}} \varepsilon_{\lambda, \lambda^{\prime}}^{(0)}(\boldsymbol{k}) c_{\boldsymbol{k}, \lambda}^{\dagger} c_{\boldsymbol{k}, \lambda^{\prime}}
$$

where $c_{\boldsymbol{k}, \lambda}^{\dagger}\left(c_{\boldsymbol{k}, \lambda}\right)$ are creation (annihilation) operators for the wave vector $\boldsymbol{k}$ and state $\lambda$ (including spin, orbital and layer degrees of freedom). Next, we incorporate the electron-electron Coulomb interaction given by

$$
V=\frac{1}{2} \sum_{\boldsymbol{k}, \boldsymbol{k}^{\prime}, \boldsymbol{q}} \sum_{\lambda, \lambda^{\prime}} \widetilde{V}_{\lambda \lambda^{\prime}}(\boldsymbol{q}) c_{\boldsymbol{k}+\boldsymbol{q}, \lambda}^{\dagger} c_{\boldsymbol{k}^{\prime}-\boldsymbol{q}, \lambda^{\prime}}^{\dagger} c_{\boldsymbol{k}^{\prime}, \lambda^{\prime}} c_{\boldsymbol{k}, \lambda},
$$

where $\widetilde{V}_{\lambda \lambda^{\prime}}(\boldsymbol{q})=\frac{2 \pi e^{2}}{\epsilon|\boldsymbol{q}|} e^{-|\boldsymbol{q}| d_{\lambda \lambda^{\prime}}}$ is the 2D Fourier transform of the real-space Coulomb interaction $\widetilde{V}_{\lambda \lambda^{\prime}}(\boldsymbol{x})=$ $\frac{e^{2}}{\epsilon \sqrt{|x|^{2}+d_{\lambda \lambda^{\prime}}^{2}}}$, and $d_{\lambda \lambda^{\prime}}$ refers to the distance between the $\lambda$ and $\lambda^{\prime}$ states.

By employing a mean-field Hartree approximation, we can reduce the full Hamiltonian $H=H_{0}+V$ to

$$
H_{\mathrm{MF}}=H_{0}+\sum_{\boldsymbol{k}, \lambda} \varepsilon_{\lambda}^{(\mathrm{H})} c_{\boldsymbol{k}, \lambda}^{\dagger} c_{\boldsymbol{k}, \lambda}
$$

where

$$
\varepsilon_{\lambda}^{(\mathrm{H})}=\sum_{\lambda^{\prime}} \widetilde{V}_{\lambda \lambda^{\prime}}(0) n_{\lambda^{\prime}}
$$

and $n_{\lambda}=\sum_{\boldsymbol{k}}\left\langle c_{\boldsymbol{k} \lambda}^{\dagger} c_{\boldsymbol{k} \lambda}\right\rangle$ [41]. The induced potential difference between the $\lambda$ and $\lambda^{\prime}$ states is given by

$$
\begin{aligned}
\varepsilon_{\lambda}^{(\mathrm{H})}-\varepsilon_{\lambda^{\prime}}^{(\mathrm{H})} & =\sum_{\lambda^{\prime \prime}}\left[\widetilde{V}_{\lambda \lambda^{\prime \prime}}(0)-\widetilde{V}_{\lambda^{\prime} \lambda^{\prime \prime}}(0)\right] n_{\lambda^{\prime \prime}} \\
& =\sum_{\lambda^{\prime \prime}} \frac{2 \pi e^{2}}{\epsilon}\left(d_{\lambda^{\prime} \lambda^{\prime \prime}}-d_{\lambda \lambda^{\prime \prime}}\right) n_{\lambda^{\prime \prime}} .
\end{aligned}
$$

Note that $\widetilde{V}_{\lambda \lambda^{\prime \prime}}(0)-\widetilde{V}_{\lambda^{\prime} \lambda^{\prime \prime}}(0)=\frac{2 \pi e^{2}}{\epsilon}\left(d_{\lambda^{\prime} \lambda^{\prime \prime}}-d_{\lambda \lambda^{\prime \prime}}\right)$ can be obtained by taking the limit $|\boldsymbol{q}|^{\epsilon} \rightarrow 0$. Therefore, the total onsite energy difference between the $\lambda$ and $\lambda^{\prime}$ states including the contribution from the Hartree potential and that from the external electric field $E_{\text {ext }}$, is given by

$$
\varepsilon_{\lambda}^{(\text {tot })}-\varepsilon_{\lambda^{\prime}}^{(\mathrm{tot})}=\varepsilon_{\lambda \lambda}^{(0)}-\varepsilon_{\lambda^{\prime} \lambda^{\prime}}^{(0)}+\varepsilon_{\lambda}^{(\mathrm{H})}-\varepsilon_{\lambda^{\prime}}^{(\mathrm{H})}+e E_{\mathrm{ext}} d_{\lambda \lambda^{\prime}} \cdot(9)
$$

Because the whole system including the top/bottom gates and the sample in-between is charge-neutral, the sum of the top-gate, bottom-gate, and sample charge densities must be zero. Thus, for the given top-gate $\left(n_{\mathrm{tg}}\right)$ and bottom-gate $\left(n_{\mathrm{bg}}\right)$ charge densities, the total carrier density, $n_{\text {tot }}=-\left(n_{\text {tg }}+n_{\text {bg }}\right)$ is induced for the sample, and the Fermi energy $\varepsilon_{\mathrm{F}}$ of the sample can be calculated from $n_{\text {tot }}$. The top-gate and bottomgate charge densities also determine the external electric field as $E_{\text {ext }}=\frac{1}{2}\left(E_{\mathrm{tg}}+E_{\mathrm{bg}}\right)$, where $E_{\mathrm{tg}}=\frac{4 \pi e}{\epsilon} n_{\mathrm{tg}}$ and
$E_{\mathrm{bg}}=-\frac{4 \pi e}{\epsilon} n_{\mathrm{bg}}$. Therefore, by solving Eqs. (8) and (9) for the given $n_{\text {tot }}\left(\right.$ or $\varepsilon_{\mathrm{F}}$ ) and $E_{\text {ext }}$, the onsite energies $\varepsilon_{\lambda}^{(\text {tot })}$ can be self-consistently obtained.

The approach we adopted above, a self-consistent Hartree method, is essentially equivalent to solving the self-consistent Poisson equation presented in $\mathrm{Li}$ et al. 42. When an external electric field is applied, the charge carriers in a few-layer BP system are redistributed in such a manner that the electrostatic energy of the system is minimized. (see 42 for details of charge distribution for various set-ups.) In this sense, the Hartree contribution consists precisely of the classical electrostatic potential generated by induced charges.

In the next section, we directly apply Eqs. (8) and (9) to few-layer BP to analyze the influence of an external electric field. For this calculation, we employ the intralayer distance $d_{\text {intra }}=2.13 \AA$ and interlayer distance $d_{\text {inter }}=3.17 \AA$. We set the dielectric constant $\epsilon=1$ for simplicity, and the choice of $\epsilon$ does not change our results qualitatively.

\section{B. Evolution of the material parameters}

Figure 3 illustrates the evolution of the energy gap parameter $\varepsilon_{\mathrm{g}}, \gamma$, effective mass $m$, and velocity $v$ under the charge neutral condition $\left(n_{\mathrm{tg}}+n_{\mathrm{tg}}=0\right)$. As the electric field (or equivalently, $n_{\mathrm{bg}}-n_{\mathrm{tg}}$ ) increases, $\gamma$ and $m$ increase, whereas $\varepsilon_{\mathrm{g}}$ and $v$ decrease. After reaching the semi-Dirac point, the parameters vary slowly with the electric field, owing to enhanced screening.

\section{OPTICAL CONDUCTIVITY}

\section{A. Kubo formula}

The Kubo formula for the optical conductivity in the non-interacting and clean limit can be expressed as 43.

$$
\begin{aligned}
\sigma_{i j}(\omega) & =-\frac{i e^{2}}{\hbar} \sum_{s, s^{\prime}} \int \frac{d^{2} k}{(2 \pi)^{2}} \frac{f_{s, \boldsymbol{k}}-f_{s^{\prime}, \boldsymbol{k}}}{\varepsilon_{s, \boldsymbol{k}}-\varepsilon_{s^{\prime}, \boldsymbol{k}}} \\
& \times \frac{M_{i}^{s s^{\prime}}(\boldsymbol{k}) M_{j}^{s^{\prime} s}(\boldsymbol{k})}{\hbar \omega+\varepsilon_{s, \boldsymbol{k}}-\varepsilon_{s^{\prime}, \boldsymbol{k}}+i 0^{+}},
\end{aligned}
$$

where $i, j=x, y, z, f_{s, \boldsymbol{k}}=1 /\left[1+e^{\left(\varepsilon_{s, \boldsymbol{k}}-\mu\right) / k_{\mathrm{B}} T}\right]$ is the Fermi distribution function for the band index $s$ and wave vector $\boldsymbol{k}, \mu$ is the chemical potential, and $M_{i}^{s s^{\prime}}(\boldsymbol{k})=$ $\left\langle s, \boldsymbol{k}\left|\hbar \hat{v}_{i}\right| s^{\prime}, \boldsymbol{k}^{\prime}\right\rangle$, with the velocity operator $\hat{v}_{i}$ obtained from the relation $\hat{v}_{i}=\frac{1}{\hbar} \frac{\partial H}{\partial k_{i}}$. In the following, we only consider the real part of the longitudinal optical conductivity in the clean limit. 

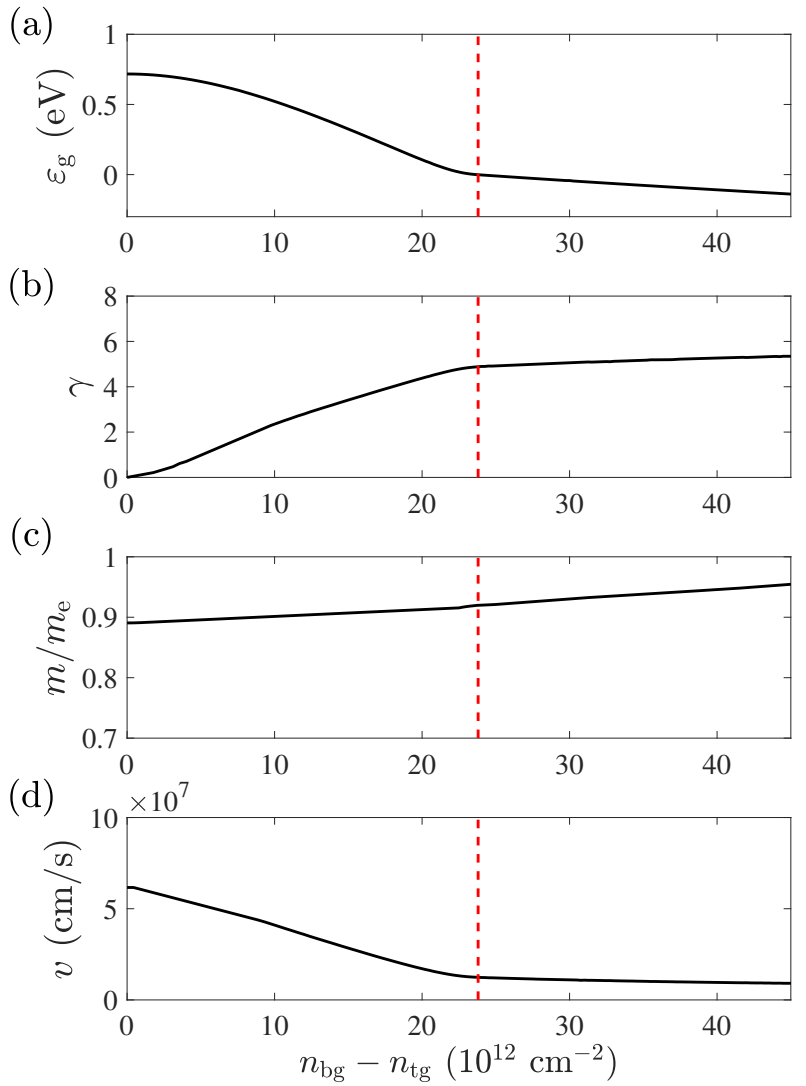

FIG. 3: Evolution of the parameters (a) $\varepsilon_{\mathrm{g}}$, (b) $\gamma$, (c) $m / m_{\mathrm{e}}$, and (d) $v$ as a function of $n_{\mathrm{bg}}-n_{\mathrm{tg}}$ under the charge neutral condition. Here, $m_{\mathrm{e}}$ is the electron mass. The red dotted line represents the semi-Dirac point corresponding to the phase boundary between the insulator phase (left side) and Dirac semimetal phase (right side).

\section{B. Optical conductivity for each phase}

In this section, we present the real part of the optical conductivity of few-layer BP for the lattice model [Eq. (1)] and continuum model [Eq. (3)]. For the lattice calculations, we self-consistently obtain the on-site energies in the presence of a perpendicular external electric field, as explained in Sec.III. Here, we focus on tetralayer $\mathrm{BP}$, and we discuss the effect of the number of layers later in Sec. V]. For the continuum model, we employ a set of parameters obtained by fitting to the lattice model near the $\Gamma$ point, for comparison.

\section{Insulator phase}

Figure 4 illustrates the calculated optical conductivities in the insulator phase for the lattice (black solid line) and corresponding continuum (red dashed line) models. The blue dotted line represents the analytic result with
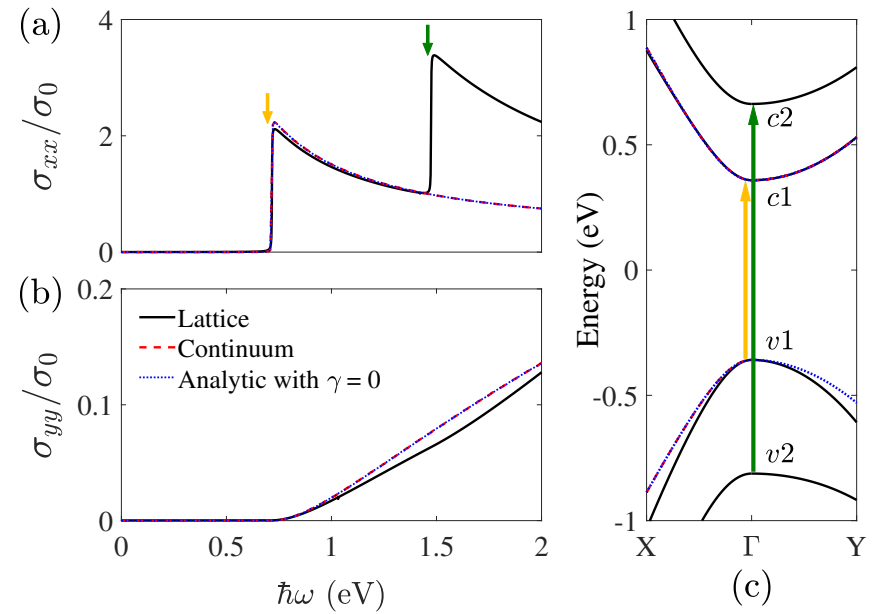

FIG. 4: Optical conductivities (a) $\sigma_{x x}$ and (b) $\sigma_{y y}$ of tetralayer $\mathrm{BP}$ in the insulator phase with zero $E_{\text {ext }}$ for the lattice model (black solid line), continuum model (red dashed line), and analytic result with $\gamma=0$ (blue dotted line). (c) The band structure of tetralayer BP in the insulator phase. Arrows indicate the interband transitions corresponding to the kink structures in $\sigma_{x x}$. Here, $\sigma_{0}=\frac{e^{2}}{4 \hbar}$, and we adopt the following parameters for the calculation: $E_{\text {ext }}=0 \mathrm{~V} / \AA$ (pristine case), $\varepsilon_{\mathrm{g}}=0.717 \mathrm{eV}, m=0.89 m_{\mathrm{e}}, \gamma=0$ and $v=6.2 \times 10^{7} \mathrm{~cm} / \mathrm{s}$.

$\gamma=0$ obtained in the vicinity of $\hbar \omega=\varepsilon_{\mathrm{g}}$ :

$$
\begin{aligned}
& \sigma_{x x}(\omega) \approx \frac{g_{\mathrm{s}} e^{2}}{4 \hbar} \sqrt{\frac{\varepsilon_{0}}{\varepsilon_{\mathrm{g}}}}\left[\frac{1}{2}-\frac{11\left(\hbar \omega-\varepsilon_{\mathrm{g}}\right)}{16 \varepsilon_{\mathrm{g}}}\right] \Theta\left(\hbar \omega-\varepsilon_{\mathrm{g}}\right)(11 \\
& \sigma_{y y}(\omega) \approx \frac{g_{\mathrm{s}} e^{2}}{4 \hbar}\left(\frac{\varepsilon_{0}}{\varepsilon_{\mathrm{g}}}\right)^{3 / 2}\left(\frac{\hbar \omega-\varepsilon_{\mathrm{g}}}{\varepsilon_{0}}\right)^{2} \Theta\left(\hbar \omega-\varepsilon_{\mathrm{g}}\right),(11 \mathrm{~b})
\end{aligned}
$$

where $\varepsilon_{0}=2 m_{y} v^{2}, g_{\mathrm{s}}=2$ accounts for spin degeneracy, and $\Theta(x)$ is the step function, with $\Theta(x)=1$ for $x>0$ and 0 otherwise.

The energy gap with size $\varepsilon_{\mathrm{g}}$ leads to zero conductivity for frequencies $\hbar \omega<\varepsilon_{\mathrm{g}}$, owing to the absence of interband transitions. At the onset of interband transitions at $\hbar \omega=\varepsilon_{\mathrm{g}}, \sigma_{x x}$ exhibits a sudden jump and then decreases linearly, while $\sigma_{y y}$ increases quadratically with an increasing frequency $\omega$.

It is also worth noting that $\sigma_{x x}$ exhibits two distinct kink structures at $\hbar \omega=0.72 \mathrm{eV}$ and $\hbar \omega=1.48 \mathrm{eV}$, which are attributed to interband transitions between states near the $\Gamma$ point, as indicated by yellow $\left(v_{1} \rightarrow c_{1}\right)$ and green $\left(v_{2} \rightarrow c_{2}\right)$ arrows in Fig. 4(c), whereas other interband transitions $\left(v_{1} \rightarrow c_{2}\right.$ and $\left.v_{2} \rightarrow c_{1}\right)$ are forbidden [17, 22. Along the zigzag direction, $\sigma_{y y}$ lacks such features because the interband transitions around the $\Gamma$ point are suppressed owing to the selection rule [21].

However, a perpendicular electric field breaks the symmetry which is responsible for the two forbidden transitions indicated by blue $\left(v_{1} \rightarrow c_{2}\right)$ and brown $\left(v_{2} \rightarrow c_{1}\right)$ arrows in Fig. 5(c). Thus these forbidden interband transitions are now allowed in $\sigma_{x x}$ [22. Note that $\mathcal{M}_{y}$ is 

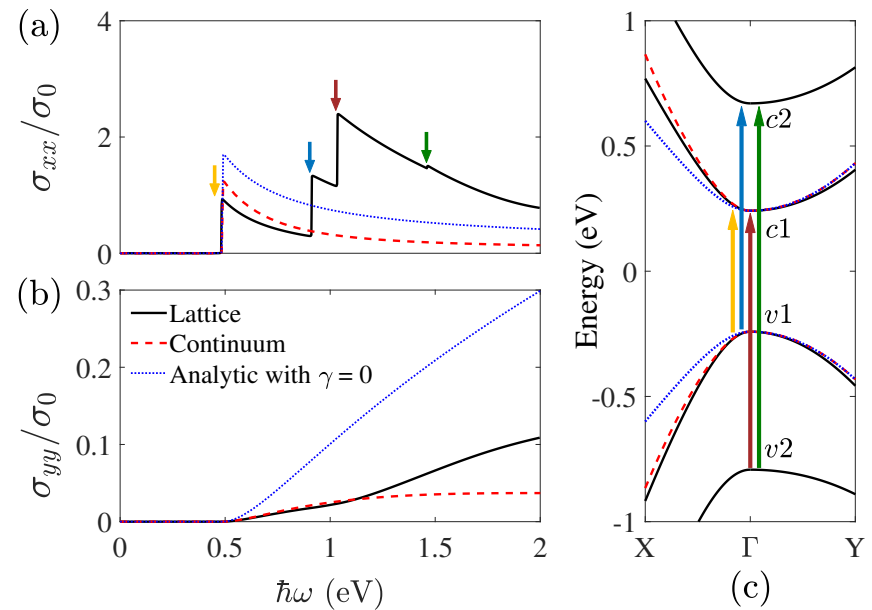

FIG. 5: Optical conductivities (a) $\sigma_{x x}$ and (b) $\sigma_{y y}$ of tetralayer $\mathrm{BP}$ in the insulator phase with finite $E_{\text {ext }}$ for the lattice model (black solid line), continuum model (red dashed line), and analytic result with $\gamma=0$ (blue dotted line). (c) The band structure of tetralayer BP in the insulator phase. Arrows indicate the interband transitions corresponding to the kink structures in $\sigma_{x x}$. Here, $\sigma_{0}=\frac{e^{2}}{4 \hbar}$, and we adopt the following parameters for the calculation: $E_{\text {ext }}=0.1 \mathrm{~V} / \AA$, $\varepsilon_{\mathrm{g}}=0.483 \mathrm{eV}, m=0.90 m_{\mathrm{e}}, \gamma=2.6$, and $v=3.8 \times 10^{7} \mathrm{~cm} / \mathrm{s}$.

still preserved in the presence of a perpendicular electric field, and thus the optical conductivity of biased few-layer $\mathrm{BP}$ exhibits suppression in $\sigma_{y y}$ near $\hbar \omega=\varepsilon_{\mathrm{g}}$. This result qualitatively agrees with recent experiments on the optical response of few-layer BP under a perpendicular electric field 223, 24. The evolution of the optical peaks with the electric field and a detailed explanation of the selection rule are given in Appendix B.

As forbidden interband transitions appear, the oscillator strength of the optical transitions, indicated by yellow $\left(v_{1} \rightarrow c_{1}\right)$ and green arrows $\left(v_{2} \rightarrow c_{2}\right)$, is reduced [Fig. 5(a)]. However, the continuum model and analytic result cannot capture this reduced oscillator strength, because they are effective two-band models at low energies, only including transitions between the two bands.

\section{Semi-Dirac point}

The zero-temperature optical conductivity with $\gamma=0$ at the semi-Dirac point $\left(\varepsilon_{\mathrm{g}}=0\right)$ is given by

$$
\begin{aligned}
& \sigma_{x x}(\omega)=g_{\mathrm{s}} \frac{e^{2}}{4 \hbar} \\
& \times\left[A_{x x}\left(\frac{|\mu|}{\varepsilon_{0}}\right)^{\frac{1}{2}} \delta(\hbar \omega)+B_{x x}\left(\frac{\omega}{\omega_{0}}\right)^{-\frac{1}{2}} \Theta\left(\omega-2\left|\omega_{\mu}\right|\right)\right], \\
& \sigma_{y y}(\omega)=g_{\mathrm{s}} \frac{e^{2}}{4 \hbar} \\
& \times\left[A_{y y}\left(\frac{|\mu|}{\varepsilon_{0}}\right)^{\frac{3}{2}} \delta(\hbar \omega)+B_{y y}\left(\frac{\omega}{\omega_{0}}\right)^{\frac{1}{2}} \Theta\left(\omega-2\left|\omega_{\mu}\right|\right)\right],
\end{aligned}
$$

where $\mu$ is the chemical potential, $\omega_{\mu}=\mu / \hbar, A_{x x}=$ $\frac{2}{3 \sqrt{\pi}} \frac{\Gamma(1 / 4)}{\Gamma(3 / 4)}, A_{y y}=\frac{48}{5 \sqrt{\pi}} \frac{\Gamma(3 / 4)}{\Gamma(1 / 4)}, B_{x x}=\frac{1}{6 \sqrt{2 \pi}} \frac{\Gamma(1 / 4)}{\Gamma(3 / 4)}$, and $B_{y y}=\frac{4 \sqrt{2}}{5 \sqrt{\pi}} \frac{\Gamma(3 / 4)}{\Gamma(1 / 4)}$. Here, $\Gamma(z)=\int_{0}^{\infty} t^{z-1} e^{-t} d t$ is the gamma function. (See Appendix A for detailed derivations.)

The first term represents intraband transitions, giving rise to the Drude peak at low frequencies. The second term represents interband transitions, which are forbidden at $\omega<2\left|\omega_{\mu}\right|$ owing to Pauli blocking. Because a finite $\mu$ simply leads to Pauli blocking for interband transitions and the Drude peak for intraband transitions, from now on we only consider the undoped case with $\mu=0$. In the undoped case, the optical conductivity at low frequencies scales as a power law, with $\sigma_{x x}(\omega) \propto \omega^{-\frac{1}{2}}$ (armchair direction) and $\sigma_{y y}(\omega) \propto \omega^{\frac{1}{2}}$ (zigzag direction), which is consistent with previous studies [44, 45].

Figure 6 illustrates the calculated optical conductivities at the semi-Dirac point for the lattice and continuum models, along with the analytic result with $\gamma=0$ [Eq. [12)]. As in the case for the insulator phase, the three results are in good agreement at low frequencies. However, as the frequency increases the analytic result begins to deviate from both the continuum and lattice results, which is particularly striking for $\sigma_{y y}$ : here, the analytic result continues to increase with the $\omega^{\frac{1}{2}}$ dependence, while the other two results monotonically decrease with the frequency. Such a deviation can be explained by the effect of the parabolic term $\gamma \frac{\hbar^{2} k_{x}^{2}}{2 m}$ in Eq. 33. Because there are both linear $\left(\hbar v k_{x}\right)$ and parabolic $\left(\gamma \frac{\hbar^{2} k_{x}^{2}}{2 m}\right)$ terms in $k_{x}$, there exists a crossover energy $\hbar \omega_{\mathrm{cr}}=\hbar v k_{x}=$ $\gamma \frac{\hbar^{2} k_{x}^{2}}{2 m}$, which is given by $\hbar \omega_{\mathrm{cr}}=\frac{2 m v^{2}}{\gamma}$. For $\omega \ll \omega_{\mathrm{cr}}$, the linear term is dominant, and the optical conductivity exhibits $\sigma_{y y} \sim \omega^{1 / 2}$, as obtained in Eq. 12 p) by neglecting the parabolic term $(\gamma=0)$. However, for $\omega \gg \omega_{\text {cr }}$ the parabolic term is dominant, and $\sigma_{y y} \sim \omega^{-1}$. The effect of $\gamma$ becomes significant as the phase changes from the insulator to the Dirac semimetal phase, as shown in Fig. 3(b). 

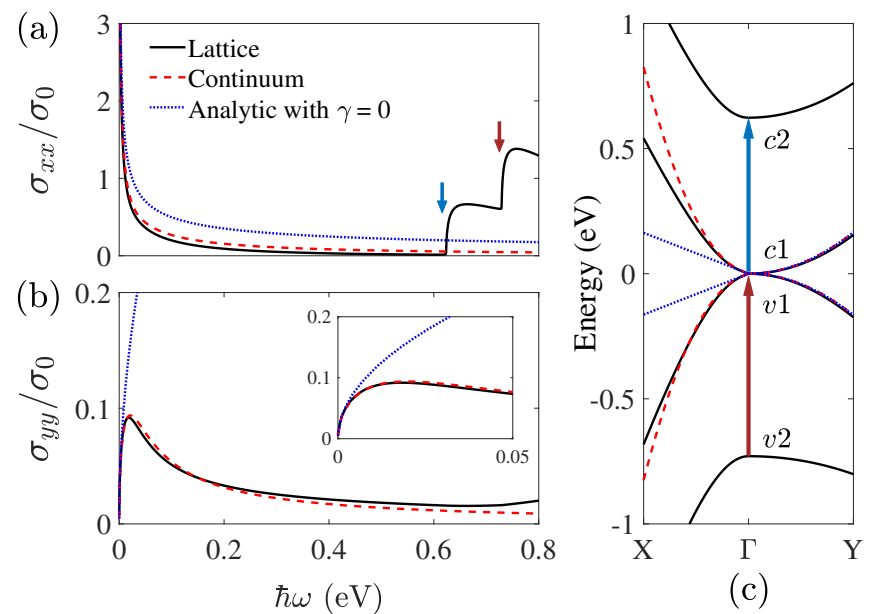

FIG. 6: Optical conductivities (a) $\sigma_{x x}$ and (b) $\sigma_{y y}$ of tetralayer $\mathrm{BP}$ at the semi-Dirac point for the lattice model (black solid line), continuum model (red dashed line), and analytic result with $\gamma=0$ (blue dotted line). (c) The band structure of tetralayer BP at the semi-Dirac point. Arrows indicate the interband transitions corresponding to the kink structures in $\sigma_{x x}$. Here, $\sigma_{0}=\frac{e^{2}}{4 \hbar}$, and we adopt the following parameters for the calculation: $E_{\text {ext }}=0.2141 \mathrm{~V} / \AA, \varepsilon_{\mathrm{g}}=0$ $\mathrm{eV}, m=0.92 m_{\mathrm{e}}, \gamma=4.9$, and $v=1.2 \times 10^{7} \mathrm{~cm} / \mathrm{s}$.

\section{Dirac semimetal phase}

The zero-temperature optical conductivity with $\gamma=0$ for the Dirac semimetal phase $\left(\varepsilon_{\mathrm{g}}<0\right)$ at low frequencies with $\mu=0$ is given by

$$
\begin{aligned}
& \sigma_{x x}(\omega) \approx 2 g_{\mathrm{s}} \frac{e^{2}}{16 \hbar} \frac{v_{x}}{v_{y}}+g_{\mathrm{s}} \frac{e^{2}}{4 \hbar} C_{x x}\left(\frac{\omega}{\omega_{0}}\right)^{2}, \\
& \sigma_{y y}(\omega) \approx 2 g_{\mathrm{s}} \frac{e^{2}}{16 \hbar} \frac{v_{y}}{v_{x}}+g_{\mathrm{s}} \frac{e^{2}}{4 \hbar} C_{y y}\left(\frac{\omega}{\omega_{0}}\right)^{2},
\end{aligned}
$$

where $v_{x}=v, v_{y}=\frac{\hbar k_{\mathrm{D}}}{m_{y}}=\sqrt{\frac{\left|\varepsilon_{\mathrm{g}}\right|}{m_{y}}}$ with $k_{\mathrm{D}}=\sqrt{\frac{m_{y}\left|\varepsilon_{\mathrm{g}}\right|}{\hbar^{2}}}$, $C_{x x}=\frac{9}{64 \sqrt{2}}\left(\frac{\varepsilon_{0}}{\left|\varepsilon_{\mathrm{g}}\right|}\right)^{5 / 2}$, and $C_{y y}=-\frac{1}{32 \sqrt{2}}\left(\frac{\varepsilon_{0}}{\left|\varepsilon_{\mathrm{g}}\right|}\right)^{3 / 2}$.

As $\varepsilon_{\mathrm{g}}$ decreases below zero, the semi-Dirac point located at the $\Gamma$ point is split into two Dirac nodes at $\boldsymbol{k}=\left(0, \pm k_{\mathrm{D}}\right)$ (see Fig. 1). Thus, the optical conductivities in the zero-frequency limit can be interpreted as the sum of the optical conductivities from the two independent 2D Dirac nodes (such as graphene) with the anisotropic in-plane velocities $v_{x}$ and $v_{y}$. (See Appendix A for detailed derivations.) Note that these velocities vary with the electric field, as shown in Fig. 3

Figure 7 illustrates the optical conductivities calculated in the Dirac semimetal phase for the lattice and continuum models, along with the analytic result obtained by assuming that $\gamma=0$ [Eq. 133$]$. If $\gamma=0$, then an optical peak in $\sigma_{x x}$ occurs at $\hbar \omega=\left|\varepsilon_{\mathrm{g}}\right|$, owing to the interband transition at the $\Gamma$ point. However, as shown in Fig. 3, $\gamma$ increases as the phase changes from
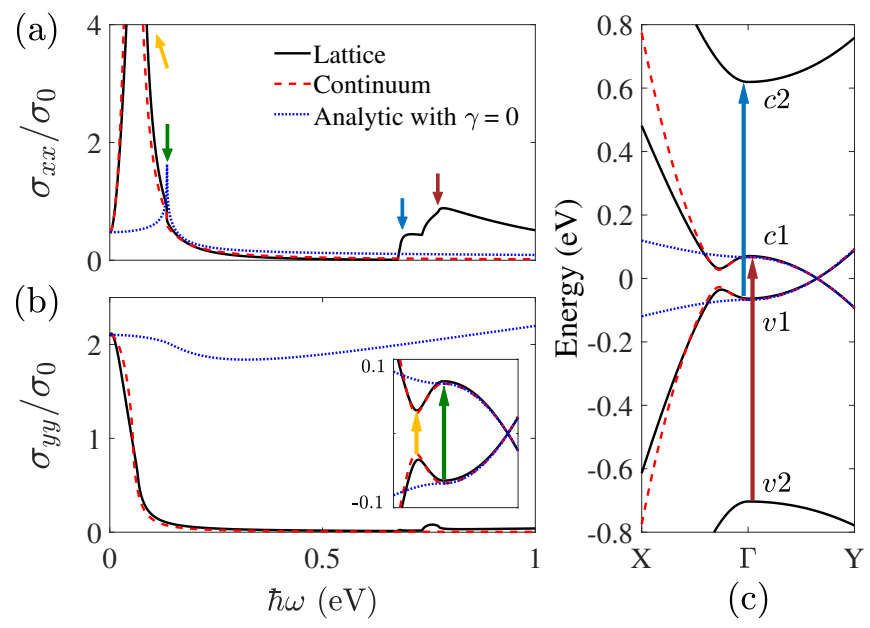

FIG. 7: Optical conductivities (a) $\sigma_{x x}$ and (b) $\sigma_{y y}$ of tetralayer BP in the Dirac semimetal phase for the lattice model (black solid line), continuum model (red dashed line), and analytic result with $\gamma=0$ (blue dotted line). (c) The band structure of tetralayer BP in the Dirac semimetal phase. Arrows indicate the interband transitions corresponding to the kink structures in $\sigma_{x x}$. Here, $\sigma_{0}=\frac{e^{2}}{4 \hbar}$ and we adopt the following parameters for the calculation: $E_{\text {ext }}=0.4 \mathrm{~V} / \AA$, $\varepsilon_{\mathrm{g}}=-0.134 \mathrm{eV}, m=0.95 m_{\mathrm{e}}, \gamma=5.2$ and $v=7.5 \times 10^{6}$ $\mathrm{cm} / \mathrm{s}$.

the insulator to the Dirac semimetal phase. Thus, it is expected that the analytic result with $\gamma=0$ will exhibit a deviation from those of the lattice and continuum models. For non-zero $\gamma$, the band structure is modified and a shifted interband transition occurs away from the $\Gamma$ point, exhibiting an optical peak at $\omega=\omega_{\mathrm{cr}} \sqrt{\frac{2\left|\varepsilon_{\mathrm{g}}\right|}{\hbar \omega_{\mathrm{cr}}}-1}$ (which is typically less than the order of $100 \mathrm{meV}$ for fewlayer BP) if $\hbar \omega_{\text {cr }}<2\left|\varepsilon_{\mathrm{g}}\right|$ (or equivalently $\gamma>\frac{m v^{2}}{\left|\varepsilon_{\mathrm{g}}\right|}$ ). For tetralayer BP, the optical peak occurs around $\hbar \omega=0.067$ $\mathrm{eV}$, as indicated by the yellow arrow in Fig. 7. The analytic result with $\gamma=0$ cannot capture this peak, because the band structure with $\gamma=0$ does not show van Hove singularities other than the $\Gamma$ point, exhibiting only one peak around $\hbar \omega=0.134 \mathrm{eV}$, as indicated by the green arrow. Kink structures in $\sigma_{x x}$ at higher frequencies are indicated by blue and brown arrows.

It is worth noting that the low-frequency optical conductivity for non-zero $\gamma$ has the same form in Eq. (13) as obtained for $\gamma=0$, but with different coefficients $C_{x x}$ and $C_{y y}$. We found that both $C_{x x}$ and $C_{y y}$ are enhanced by increasing $\gamma$, whereas $v_{x}$ and $v_{y}$ do not change with $\gamma$, giving the same optical conductivity in the zerofrequency limit irrespective of $\gamma$.

The optical conductivity along the zigzag direction $\sigma_{y y}$ does not exhibit any peaks in $\sigma_{x x}$, because the vanishing matrix elements forbid such transitions. As the frequency increases, a discrepancy in $\sigma_{y y}$ between the analytic result and the results of the two other models becomes significant. Both the lattice and continuum model results 

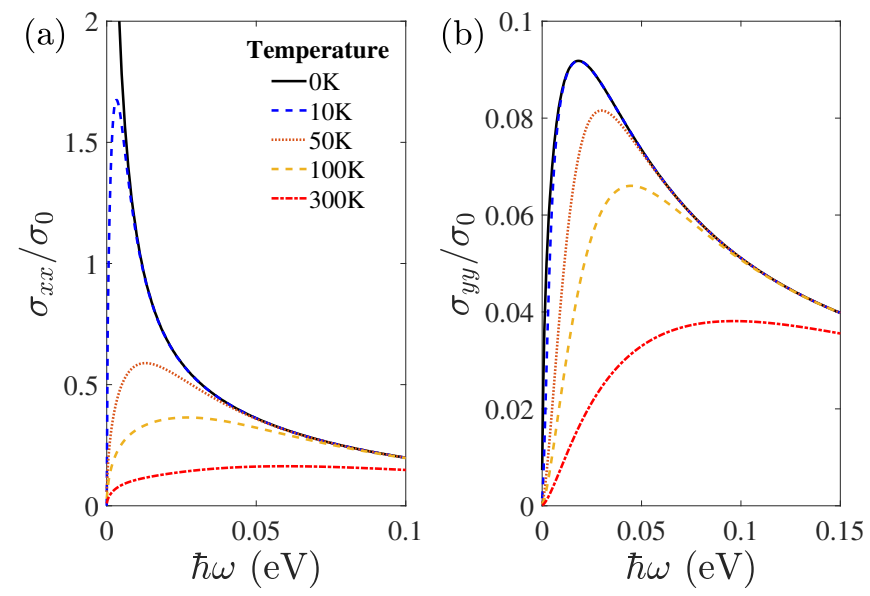

FIG. 8: Optical conductivities (a) $\sigma_{x x}$ and (b) $\sigma_{y y}$ of tetralayer BP obtained using the lattice model at the semiDirac point for various temperatures with $\mu=0$. Here, we adopt the same parameters as in Fig. 6 for the calculation.

decrease, whereas the analytic result increases, owing to the absence of the parabolic term $\gamma \frac{\hbar^{2} k_{x}^{2}}{2 m}$.

\section{Finite temperature effect}

So far, we have focused on the zero temperature case. In this section, we analyze the effect of a finite temperature on the optical conductivity at low frequencies. If conduction and valence bands are symmetric in a twoband model, then the optical conductivity at finite temperature is reduced to a compact form as follows (see Appendix A for details):

$$
\sigma_{i i}(\omega, T, \mu)=A(\omega, T, \mu) \sigma_{i i}(\omega, T=0, \mu=0),
$$

where

$$
A(\omega, T, \mu)=\frac{\sinh \left(\frac{\hbar \omega}{2 k_{\mathrm{B}} T}\right)}{\cosh \left(\frac{\hbar \omega}{2 k_{\mathrm{B}} T}\right)+\cosh \left(\frac{\mu}{k_{\mathrm{B}} T}\right)} .
$$

Note that $A(\omega, T=0, \mu)=\Theta(\hbar \omega-2|\mu|)$ for $T=0$, reproducing the zero-temperature result with Pauli blocking. Furthermore, note that for $\mu=0, A(\omega, T, \mu=0)=$ $\tanh \left(\frac{\hbar \omega}{4 k_{\mathrm{B}} T}\right)$.

Figure 8 illustrates the optical conductivities for the semi-Dirac point calculated at finite temperature with $\mu=0$. At zero temperature, the low-frequency powerlaw of the optical conductivities at the semi-Dirac point is given by $\sigma_{x x} \sim \omega^{1 / 2}$ and $\sigma_{y y} \sim \omega^{-1 / 2}$. At finite temperature, the temperature factor $A(\omega, T, \mu=0)=$ $\tanh \left(\frac{\hbar \omega}{4 k_{\mathrm{B}} T}\right)$ is multiplied, and so the power-law is modified to $\sigma_{x x} \sim \frac{\omega^{3 / 2}}{T}$ and $\sigma_{y y} \sim \frac{\omega^{1 / 2}}{T}$ for $k_{\mathrm{B}} T \gg \hbar \omega$. Here, we used $\tanh (x) \approx x$ for small $x$. Similarly, in the insulator and Dirac semimetal phases, the optical conductivity at finite temperature exhibits a modified power-law at low frequencies.

\section{DISCUSSION AND SUMMARY}

The present calculations are performed for tetralayer BP. As the number of layers increases, the electronic structure of few-layer BP and the corresponding optical conductivities change. Figure 9(a) illustrates the evolution of the first and second optical peaks in the insulator phase with $E_{\text {ext }}=0$ and at the semi-Dirac phase as the number of layers increases, showing that the peak positions decrease with the number of layers. We also demonstrate how the parameter $\gamma$ evolves as the number of layers increases in Fig. (9)(b). For the insulator phase with $E_{\text {ext }}=0, \gamma$ remains around 0 regardless of the number of layers, whereas at the semi-Dirac point $\gamma$ decreases from 5.4 for three layers to 4.3 for eight layers of BP.

As the Fermi energy moves away from zero, the intraband contribution to the optical conductivity arises at low frequencies. Figure 10 shows the Drude weight for each phase. As the Fermi energy increases, the Drude weight increases and exhibits kink structures at the van Hove singularities. These features can be observed more
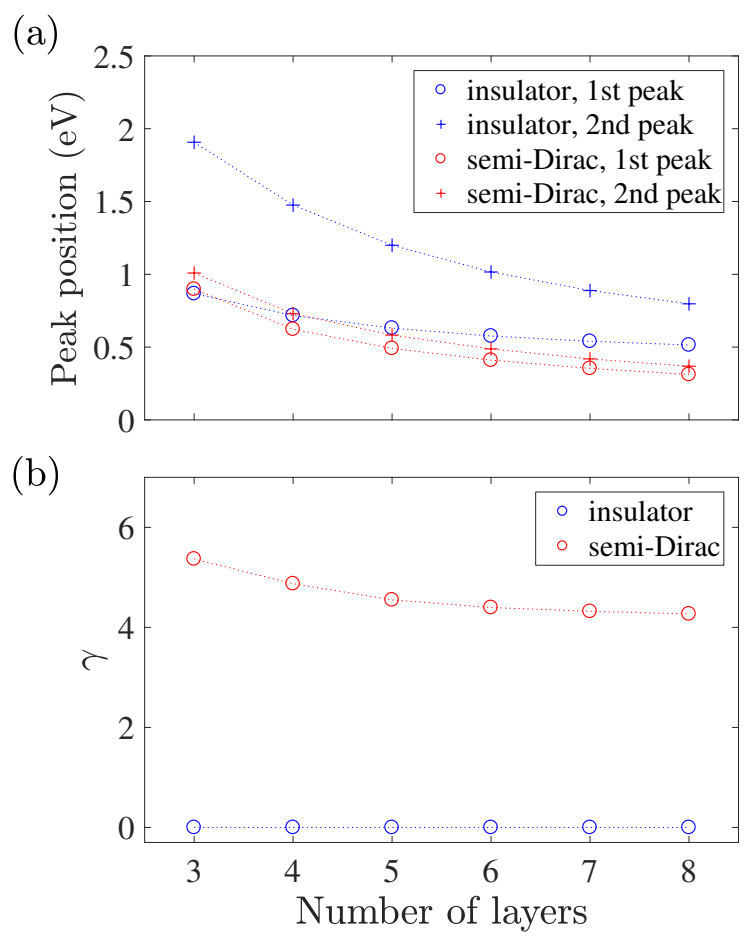

FIG. 9: (a) First and second optical peaks as a function of the number of layers in the insulator phase with $E_{\text {ext }}=0$ and the semi-Dirac point. (b) Evolution of the parameter $\gamma$ in the insulator phase with $E_{\text {ext }}=0$ and the semi-Dirac point as a function of the number of layers. 
(a)

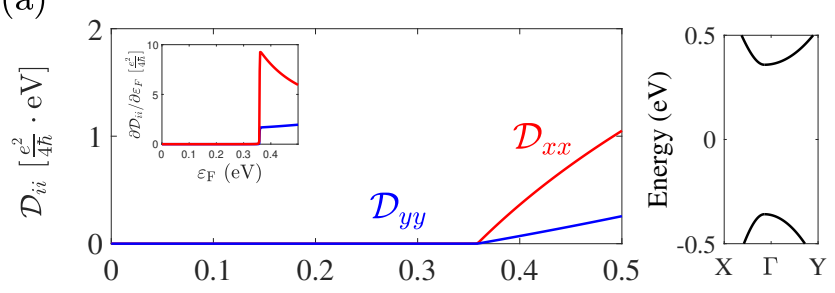

(b)
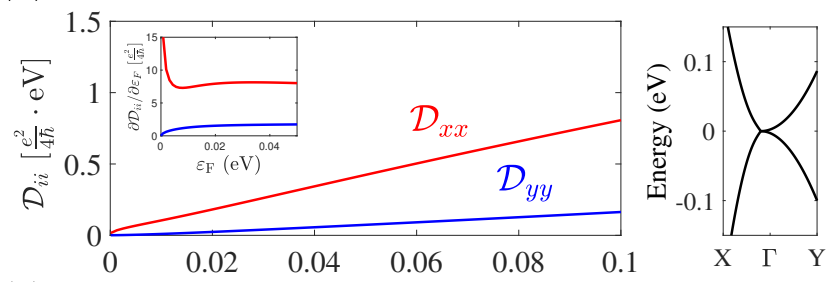

(c)

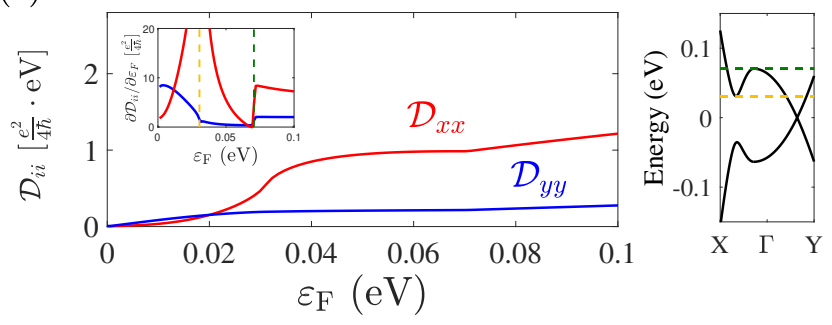

FIG. 10: Drude weight as a function of Fermi energy for the lattice model in the (a) insulator phase, (b) semi-Dirac point and (c) Dirac semimetal phase. The right panels show the corresponding energy dispersions. The Drude weight is defined to be $\sigma_{i i}=\mathcal{D}_{i i} \delta(\hbar \omega)$. The insets show the derivatives of the Drude weight.

clearly in the derivatives of the Drude weight with respect to the Fermi energy, as shown in the insets of Fig. 10.(c).
Note that in the Dirac semimetal phase, the derivative of the Drude weight exhibits discontinuities at the van Hove singularities, indicated by yellow and green arrows.

In summary, we have studied the optical conductivity of a biased few-layer BP in each phase and at the corresponding transition points. In particular, we focused on the low-energy characteristic frequency dependence, which can be utilized as an experimental fingerprint. We analytically obtained the scaling law for the optical conductivity at low frequencies, and verified this using the corresponding full lattice model for few-layer BP. Beyond the low frequency regime, the analytic result exhibits a deviation from the result calculated based on the lattice model. We systematically analyzed the role of the parabolic term $\gamma \frac{\hbar^{2} k_{x}^{2}}{2 m} \sigma_{z}$ in the optical conductivity. The parameter $\gamma$, which characterizes the contribution of the parabolic term, varies with the external electric field strength, and becomes significant as the phase changes from the insulator to the Dirac semimetal phase. At the semi-Dirac point, it was revealed that this parabolic term gives rise to a crossover frequency beyond which the low frequency scaling law $\left(\sigma_{x x} \sim \omega^{-1 / 2}, \sigma_{y y} \sim \omega^{1 / 2}\right)$ is no longer valid. In the Dirac semimetal phase, the dominant low-frequency interband transition is shifted, owing to the band distortion associated with non-zero $\gamma$.

\section{Acknowledgments}

J.J. and H.M. were supported by the NRF grant funded by the Korea government (MSIT) (No. 2018R1A2B6007837) and Creative-Pioneering Researchers Program through Seoul National University. S.A. was supported by IBS-R009-D1 (G1, G2, G3, Y1).

\section{Appendix A: Analytic expressions of optical conductivity for each phase}

In the following, we present detailed derivations of the optical conductivities for few-layer BP using the Kubo formula [Eq. [10] in the main text]. Note that for $M_{i}^{s s^{\prime}}(\boldsymbol{k})=\left\langle s, \boldsymbol{k}\left|\hbar \hat{v}_{i}\right| s^{\prime}, \boldsymbol{k}^{\prime}\right\rangle, M_{i}^{s s^{\prime}}(\boldsymbol{k}) M_{i}^{s^{\prime} s}(\boldsymbol{k})=\left|M_{i}^{s s^{\prime}}(\boldsymbol{k})\right|^{2}(i=x, y)$ is always real. Thus, the intraband and interband contributions to the real part of the longitudinal conductivity in the clean limit are given by

$$
\sigma_{i i}^{\text {intra }}(\omega)=-g_{\mathrm{s}} \frac{\pi e^{2}}{\hbar} \int \frac{d^{2} k}{(2 \pi)^{2}} \sum_{s= \pm} \frac{\partial f_{s, \boldsymbol{k}}}{\partial \varepsilon_{s, \boldsymbol{k}}}\left|M_{i}^{s s}(\boldsymbol{k})\right|^{2} \delta(\hbar \omega)
$$

and

$$
\sigma_{i i}^{\text {inter }}(\omega)=-g_{\mathrm{s}} \frac{\pi e^{2}}{\hbar} \int \frac{d^{2} k}{(2 \pi)^{2}} \frac{f_{+, \boldsymbol{k}}-f_{-, \boldsymbol{k}}}{\varepsilon_{+, \boldsymbol{k}}-\varepsilon_{-, \boldsymbol{k}}}\left|M_{i}^{+-}(\boldsymbol{k})\right|^{2} \delta\left(\hbar \omega+\varepsilon_{-, \boldsymbol{k}}-\varepsilon_{+, \boldsymbol{k}}\right)
$$

at positive frequencies $(\omega>0)$. Using this formula, it is straightforward to obtain the longitudinal optical conductivity for few-layer BP.

The model Hamiltonian for few-layer BP in Eq. (3) in the main text can be simplified by introducing dimensionless 
parameters, and reduces to

$$
H=\varepsilon_{0}\left[\widetilde{k}_{x} \sigma_{y}+\left(\frac{1}{2} \widetilde{\varepsilon}_{\mathrm{g}}+\widetilde{k}_{y}^{2}+\gamma \widetilde{k}_{x}^{2}\right) \sigma_{z}\right],
$$

where $\widetilde{k}_{x, y}=k_{x, y} / k_{0}$ are dimensionless momenta $k_{0}=\frac{2 m v}{\hbar}, \varepsilon_{0}=2 m v^{2}, \widetilde{\varepsilon}_{\mathrm{g}}=\varepsilon_{\mathrm{g}} / \varepsilon_{0}$, and the parameter $\gamma$ represents the ratio of the effective mass along the zigzag direction to that in the armchair direction. In the following, we simplify the continuum Hamiltonian [Eq. A3]] by setting $\gamma=0$, which allows an analytic form of the optical conductivity to be obtained.

\section{Intraband conductivity}

From Eq. A1, the intraband conductivity is expressed as

$$
\begin{aligned}
\sigma_{i i}^{\text {intra }}(\omega) & =-g_{\mathrm{s}} \frac{\pi e^{2}}{\hbar} \int \frac{d^{2} k}{(2 \pi)^{2}}\left(\frac{\partial f_{+, \boldsymbol{k}}}{\partial \varepsilon_{+, \boldsymbol{k}}}\left|M_{i}^{++}(\boldsymbol{k})\right|^{2}+\frac{\partial f_{-, \boldsymbol{k}}}{\partial \varepsilon_{-, \boldsymbol{k}}}\left|M_{i}^{--}(\boldsymbol{k})\right|^{2}\right) \delta(\hbar \omega) \\
& =-g_{\mathrm{s}} \frac{\pi e^{2}}{\hbar} \int \frac{d^{2} k}{(2 \pi)^{2}}\left(\frac{\partial f_{+, \boldsymbol{k}}}{\partial \varepsilon_{+, \boldsymbol{k}}}+\frac{\partial f_{-, \boldsymbol{k}}}{\partial \varepsilon_{-, \boldsymbol{k}}}\right)\left|M_{i}^{\mathrm{intra}}(\boldsymbol{k})\right|^{2} \delta(\hbar \omega),
\end{aligned}
$$

where the intraband matrix elements are given by

$$
\begin{aligned}
\left|M_{x}^{\text {intra }}(\boldsymbol{k})\right|^{2} & =\left|M_{x}^{++}(\boldsymbol{k})\right|^{2}=\left|M_{x}^{--}(\boldsymbol{k})\right|^{2}=\frac{\varepsilon_{0}^{2} \sin ^{2} \psi}{k_{0}^{2}}, \\
\left|M_{y}^{\text {intra }}(\boldsymbol{k})\right|^{2} & =\left|M_{y}^{++}(\boldsymbol{k})\right|^{2}=\left|M_{y}^{--}(\boldsymbol{k})\right|^{2}=\frac{4 \varepsilon_{0}^{2} \widetilde{k}_{y}^{2} \cos ^{2} \psi}{k_{0}^{2}},
\end{aligned}
$$

and $\psi=\tan ^{-1}\left[\widetilde{k}_{x} /\left(\widetilde{\varepsilon}_{\mathrm{g}} / 2+\widetilde{k}_{y}^{2}\right)\right]$.

The intraband optical conductivity in Eq. A4 can be rewritten as

$$
\begin{aligned}
& \frac{\sigma_{x x}^{\text {intra }}}{\sigma_{0}}=-g_{\mathrm{s}} \frac{1}{\pi} \int d^{2} \widetilde{k}\left(\frac{\partial f_{+, \boldsymbol{k}}}{\partial \widetilde{\varepsilon}_{\boldsymbol{k}}}+\frac{\partial f_{-, \boldsymbol{k}}}{\partial\left(-\widetilde{\varepsilon}_{\boldsymbol{k}}\right)}\right) \frac{\widetilde{k}_{x}^{2}}{\widetilde{\varepsilon}_{\boldsymbol{k}}^{2}} \delta\left(\frac{\hbar \omega}{\varepsilon_{0}}\right), \\
& \frac{\sigma_{y y}^{\text {intra }}}{\sigma_{0}}=-g_{\mathrm{s}} \frac{4}{\pi} \int d^{2} \widetilde{k}\left(\frac{\partial f_{+, \boldsymbol{k}}}{\partial \widetilde{\varepsilon}_{\boldsymbol{k}}}+\frac{\partial f_{-, \boldsymbol{k}}}{\partial\left(-\widetilde{\varepsilon}_{\boldsymbol{k}}\right)}\right) \frac{\widetilde{k}_{y}^{2}\left(\widetilde{\varepsilon}_{\mathrm{g}} / 2+\widetilde{k}_{y}^{2}\right)^{2}}{\widetilde{\varepsilon}_{\boldsymbol{k}}^{2}} \delta\left(\frac{\hbar \omega}{\varepsilon_{0}}\right),
\end{aligned}
$$

where $\sigma_{0}=e^{2} /(4 \hbar), \widetilde{\varepsilon}_{\boldsymbol{k}}=\varepsilon_{+}(\boldsymbol{k}) / \varepsilon_{0}=\sqrt{\left(\frac{1}{2} \widetilde{\varepsilon}_{\mathrm{g}}+\widetilde{k}_{x}^{2}\right)^{2}+\widetilde{k}_{y}^{2}}$ and $g_{\mathrm{s}}=2$ is the spin degeneracy.

At zero temperature, $f_{ \pm, \boldsymbol{k}}=\Theta\left(\mu-\varepsilon_{ \pm}(\boldsymbol{k})\right)$ and so $\lim _{T \rightarrow 0}\left(-\frac{\partial f_{ \pm, \boldsymbol{k}}}{\partial \varepsilon_{ \pm, \boldsymbol{k}}}\right)=\delta\left(\mu-\varepsilon_{ \pm}(\boldsymbol{k})\right)$. By using the relation $\delta(f(x))=$ $\sum_{i} \frac{\delta\left(x-x_{i}\right)}{\left|f^{\prime}\left(x_{i}\right)\right|}$, where $f\left(x_{i}\right)=0$, the intraband optical conductivity at zero temperature is given by

$$
\begin{aligned}
\frac{\sigma_{x x}^{\text {intra }}}{\sigma_{0}} & =g_{\mathrm{s}} \frac{1}{\pi} \int_{-\infty}^{\infty} \int_{-\infty}^{\infty} d \widetilde{k}_{x} d \widetilde{k}_{y}\left[\frac{\widetilde{k}_{x}}{\sqrt{\left(\frac{1}{2} \widetilde{\varepsilon}_{\mathrm{g}}+\widetilde{k}_{y}^{2}\right)^{2}+\widetilde{k}_{x}^{2}}}\right]^{2} \delta\left(\left(\frac{|\mu|}{\varepsilon_{0}}\right)-\sqrt{\left(\frac{1}{2} \widetilde{\varepsilon}_{\mathrm{g}}+\widetilde{k}_{y}^{2}\right)^{2}+\widetilde{k}_{x}^{2}}\right) \delta\left(\frac{\hbar \omega}{\varepsilon_{0}}\right), \\
& =g_{\mathrm{s}} \frac{4}{\pi} \frac{1}{\left(|\mu| / \varepsilon_{0}\right)} \int d \widetilde{k}_{y} \sqrt{\left(\frac{|\mu|}{\varepsilon_{0}}\right)^{2}-\left(\frac{1}{2} \widetilde{\varepsilon}_{\mathrm{g}}+\widetilde{k}_{y}^{2}\right)^{2}} \delta\left(\frac{\hbar \omega}{\varepsilon_{0}}\right), \\
\frac{\sigma_{y y}^{\text {intra }}}{\sigma_{0}} & =g_{\mathrm{s}} \frac{4}{\pi} \int_{-\infty}^{\infty} \int_{-\infty}^{\infty} d \widetilde{k}_{x} d \widetilde{k}_{y}\left[\frac{\widetilde{k}_{y}\left(\frac{1}{2} \widetilde{\varepsilon}_{\mathrm{g}}+\widetilde{k}_{y}^{2}\right)}{\sqrt{\left(\frac{1}{2} \widetilde{\varepsilon}_{\mathrm{g}}+\widetilde{k}_{y}^{2}\right)^{2}+\widetilde{k}_{x}^{2}}}\right]^{2} \delta\left(\left(\frac{|\mu|}{\varepsilon_{0}}\right)-\sqrt{\left(\frac{1}{2} \widetilde{\varepsilon}_{\mathrm{g}}+\widetilde{k}_{y}^{2}\right)^{2}+\widetilde{k}_{x}^{2}}\right) \delta\left(\frac{\hbar \omega}{\varepsilon_{0}}\right) . \\
& =g_{\mathrm{s}} \frac{16}{\pi} \frac{1}{\left(|\mu| / \varepsilon_{0}\right)} \int d \widetilde{k}_{y} \frac{\widetilde{k}_{y}^{2}\left(\frac{1}{2} \widetilde{\varepsilon}_{\mathrm{g}}+\widetilde{k}_{y}^{2}\right)^{2}}{\sqrt{\left(\frac{|\mu|}{\varepsilon_{0}}\right)^{2}-\left(\frac{1}{2} \widetilde{\varepsilon}_{\mathrm{g}}+\widetilde{k}_{y}^{2}\right)^{2}}} \delta\left(\frac{\hbar \omega}{\varepsilon_{0}}\right),
\end{aligned}
$$


First, consider the semi-Dirac case $\left(\varepsilon_{\mathrm{g}}=0\right)$. By performing the integration with $\varepsilon_{\mathrm{g}}=0$, we obtain

$$
\begin{aligned}
& \frac{\sigma_{x x}^{\text {intra }}}{\sigma_{0}}=\left[\frac{4}{3 \sqrt{\pi}} \frac{\Gamma(1 / 4)}{\Gamma(3 / 4)}\right]\left(\frac{|\mu|}{\varepsilon_{0}}\right)^{\frac{1}{2}} \delta\left(\frac{\hbar \omega}{\varepsilon_{0}}\right), \\
& \frac{\sigma_{y y}^{\text {intra }}}{\sigma_{0}}=\left[\frac{96}{5 \sqrt{\pi}} \frac{\Gamma(3 / 4)}{\Gamma(1 / 4)}\right]\left(\frac{|\mu|}{\varepsilon_{0}}\right)^{\frac{3}{2}} \delta\left(\frac{\hbar \omega}{\varepsilon_{0}}\right),
\end{aligned}
$$

Here, $\Gamma(z)=\int_{0}^{\infty} t^{z-1} e^{-t} d t$ is the gamma function.

For the insulator phase, the Drude weight defined to be $\sigma_{i i}=\mathcal{D}_{i i} \delta(\hbar \omega)$ in the vicinity of $\mu=\varepsilon_{\mathrm{g}}$, is given by

$$
\begin{aligned}
& \frac{\mathcal{D}_{x x}}{\mathcal{D}_{0}} \approx \mathcal{B}_{x}^{(0)}+\mathcal{B}_{x}^{(1)}\left(\frac{\mu-\varepsilon_{\mathrm{g}}}{\varepsilon_{0}}\right)+O\left(\mu^{2}\right), \\
& \frac{\mathcal{D}_{y y}}{\mathcal{D}_{0}} \approx \mathcal{B}_{y}^{(0)}+\mathcal{B}_{y}^{(1)}\left(\frac{\mu-\varepsilon_{\mathrm{g}}}{\varepsilon_{0}}\right)+O\left(\mu^{2}\right),
\end{aligned}
$$

where $\mathcal{D}_{0}=\frac{e^{2} \varepsilon_{0}}{4 \hbar}, \mathcal{B}_{x}^{(0)}=\frac{4 \sqrt{2}}{3 \pi}\left[-2 \mathrm{E}\left(\frac{1}{4}\right)+3 \mathrm{~K}\left(\frac{1}{4}\right)\right] \sqrt{\widetilde{\varepsilon}_{\mathrm{g}}} \approx 1.274 \sqrt{\widetilde{\varepsilon}_{\mathrm{g}}}, \mathcal{B}_{x}^{(1)}=\frac{8 \sqrt{2}}{3 \pi} \mathrm{E}\left(\frac{1}{4}\right) \frac{1}{\sqrt{\widetilde{\varepsilon}_{\mathrm{g}}}} \approx 1.762 \frac{1}{\sqrt{\widetilde{\varepsilon}_{\mathrm{g}}}}, \mathcal{B}_{y}^{(0)}=$ $\frac{32 \sqrt{2}}{15 \pi}\left[17 \mathrm{E}\left(\frac{1}{4}\right)-12 \mathrm{~K}\left(\frac{1}{4}\right)\right] \widetilde{\varepsilon}_{\mathrm{g}}^{\frac{3}{2}} \approx 4.531 \widetilde{\varepsilon}_{\mathrm{g}}^{\frac{3}{2}}$, and $\mathcal{B}_{y}^{(1)}=\frac{64 \sqrt{2}}{15 \pi}\left[14 \mathrm{E}\left(\frac{1}{4}\right)-9 \mathrm{~K}\left(\frac{1}{4}\right)\right] \widetilde{\varepsilon}_{\mathrm{g}}^{\frac{1}{2}} \approx 10.319 \widetilde{\varepsilon}_{\mathrm{g}}^{\frac{1}{2}}$.

For the Dirac semimetal phase $\left(\varepsilon_{\mathrm{g}}<0\right)$, the Drude weight at low Fermi energy is given by

$$
\begin{aligned}
& \frac{\mathcal{D}_{x x}}{\mathcal{D}_{0}} \approx \mathcal{C}_{x}^{(0)}+\mathcal{C}_{x}^{(1)}\left(\frac{\mu-\left|\varepsilon_{\mathrm{g}}\right|}{\varepsilon_{0}}\right)+O\left(\mu^{2}\right), \\
& \frac{\mathcal{D}_{y y}}{\mathcal{D}_{0}} \approx \mathcal{C}_{y}^{(0)}+\mathcal{C}_{y}^{(1)}\left(\frac{\mu-\left|\varepsilon_{\mathrm{g}}\right|}{\varepsilon_{0}}\right)+O\left(\mu^{2}\right),
\end{aligned}
$$

where $\mathcal{C}_{x}^{(0)}=\frac{4 \sqrt{2}}{3 \pi}\left[2 \mathrm{E}\left(\frac{3}{4}\right)+\mathrm{K}\left(\frac{3}{4}\right)\right] \sqrt{\mid \widetilde{\varepsilon_{\mathrm{g}} \mid}} \approx 2.748 \sqrt{\left|\widetilde{\varepsilon}_{\mathrm{g}}\right|}, \mathcal{C}_{x}^{(1)}=\frac{8 \sqrt{2}}{3 \pi}\left[-\mathrm{E}\left(\frac{3}{4}\right)+\mathrm{K}\left(\frac{3}{4}\right)\right] \frac{1}{\sqrt{\left|\widetilde{\varepsilon}_{\mathrm{g}}\right|}} \approx 1.762 \frac{1}{\sqrt{\left|\widetilde{\varepsilon}_{\mathrm{g}}\right|}}, \mathcal{C}_{y}^{(0)}=$ $\frac{32 \sqrt{2}}{15 \pi}\left[17 \mathrm{E}\left(\frac{3}{4}\right)-5 \mathrm{~K}\left(\frac{3}{4}\right)\right]\left|\widetilde{\varepsilon}_{\mathrm{g}}\right|^{\frac{3}{2}} \approx 9.416\left|\widetilde{\varepsilon}_{\mathrm{g}}\right|^{\frac{3}{2}}$, and $\mathcal{C}_{y}^{(1)}=\frac{64 \sqrt{2}}{15 \pi}\left[14 \mathrm{E}\left(\frac{3}{4}\right)-5 \mathrm{~K}\left(\frac{3}{4}\right)\right]\left|\widetilde{\varepsilon}_{\mathrm{g}}\right|^{\frac{1}{2}} \approx 11.855\left|\widetilde{\varepsilon}_{\mathrm{g}}\right|^{\frac{1}{2}}$.

At low densities (or Fermi energy), these analytic results are in good agreement with the results obtained using the lattice model (see Fig. 10 in the main text). However, as the Fermi energy increases the analytic results deviate from the lattice results, especially in the Dirac semimetal phase, because the effect of the parabolic term $\gamma \frac{\hbar^{2} k_{x}^{2}}{2 m}$ in Eq. 3 . becomes significant.

\section{Interband conductivity}

From Eq. A2, the interband conductivity is given by

$$
\sigma_{i i}^{\text {inter }}(\omega)=-g_{\mathrm{s}} \frac{\pi e^{2}}{\hbar} \int \frac{d^{2} k}{(2 \pi)^{2}} \frac{f_{+, \boldsymbol{k}}-f_{-, \boldsymbol{k}}}{\varepsilon_{+, \boldsymbol{k}}-\varepsilon_{-, \boldsymbol{k}}}\left|M_{i}^{+-}(\boldsymbol{k})\right|^{2} \delta\left(\hbar \omega+\varepsilon_{-, \boldsymbol{k}}-\varepsilon_{+, \boldsymbol{k}}\right) .
$$

In the presence of an electron-hole symmetry $\left(\varepsilon_{+, \boldsymbol{k}}=-\varepsilon_{-, \boldsymbol{k}}\right)$, we can conveniently factor out the temperature dependence into a single coefficient:

$$
\begin{aligned}
\sigma_{i i}^{\text {inter }}(\omega) & =-g_{\mathrm{s}} \frac{\pi e^{2}}{\hbar} \int \frac{d^{2} k}{(2 \pi)^{2}} \frac{f_{+, \boldsymbol{k}}-f_{-, \boldsymbol{k}}}{\varepsilon_{+, \boldsymbol{k}}-\varepsilon_{-, \boldsymbol{k}}}\left|M_{i}^{+-}(\boldsymbol{k})\right|^{2} \delta\left(\hbar \omega+\varepsilon_{-, \boldsymbol{k}}-\varepsilon_{+, \boldsymbol{k}}\right) \\
& =-g_{\mathrm{s}} \frac{\pi e^{2}}{\hbar} \int \frac{d^{2} k}{(2 \pi)^{2}} \frac{f\left(\varepsilon_{+, \boldsymbol{k}}\right)-f\left(-\varepsilon_{+, \boldsymbol{k}}\right)}{2 \varepsilon_{+, \boldsymbol{k}}}\left|M_{i}^{+-}(\boldsymbol{k})\right|^{2} \delta\left(\hbar \omega-2 \varepsilon_{+, \boldsymbol{k}}\right) \\
& =\left[f\left(-\frac{\hbar \omega}{2}\right)-f\left(\frac{\hbar \omega}{2}\right)\right]\left[g_{\mathrm{s}} \frac{\pi e^{2}}{\hbar} \int \frac{d^{2} k}{(2 \pi)^{2}} \frac{\left|M_{i}^{+-}(\boldsymbol{k})\right|^{2}}{2 \varepsilon_{+, \boldsymbol{k}}} \delta\left(\hbar \omega-2 \varepsilon_{+, \boldsymbol{k}}\right)\right] \\
& =A(\omega, T, \mu) \sigma_{i i}^{\text {inter }}(\omega, T=0, \mu=0),
\end{aligned}
$$

where

$$
A(\omega, T, \mu)=f\left(-\frac{\hbar \omega}{2}\right)-f\left(\frac{\hbar \omega}{2}\right)=\frac{\sinh (\beta \hbar \omega / 2)}{\cosh (\beta \hbar \omega / 2)+\cosh (\beta \mu)}
$$


Note that $A(\omega, T, \mu)$ absorbs all the dependence on the temperature and the chemical potential. In the following, we use $\sigma_{i i}^{\text {inter }}(\omega)$ to refer to $\sigma_{i i}^{\text {inter }}(\omega, T=0, \mu=0)$ for simplicity.

With the interband matrix elements given by

$$
\begin{aligned}
\left|M_{x}^{\text {inter }}(\boldsymbol{k})\right|^{2} & =M_{x}^{+-}(\boldsymbol{k}) M_{x}^{-+}(\boldsymbol{k})=\frac{\varepsilon_{0}^{2} \cos ^{2} \psi}{k_{0}^{2}} \\
\left|M_{y}^{\text {inter }}(\boldsymbol{k})\right|^{2} & =M_{y}^{+-}(\boldsymbol{k}) M_{y}^{-+}(\boldsymbol{k})=\frac{4 \varepsilon_{0}^{2} \widetilde{k}_{y}^{2} \sin ^{2} \psi}{k_{0}^{2}},
\end{aligned}
$$

where $\psi=\tan ^{-1}\left[\widetilde{k}_{x} /\left(\widetilde{\varepsilon}_{\mathrm{g}} / 2+\widetilde{k}_{y}^{2}\right)\right]$, we can rewrite the interband conductivity as

$$
\begin{aligned}
& \frac{\sigma_{x x}^{\text {inter }}}{\sigma_{0}}=g_{\mathrm{s}} \frac{\pi e^{2}}{\hbar} \int \frac{d^{2} k}{(2 \pi)^{2}} \frac{\left|M_{x}^{+-}(\boldsymbol{k})\right|^{2}}{2 \varepsilon_{+, k}} \delta\left(\hbar \omega-2 \varepsilon_{+, \boldsymbol{k}}\right) \\
& =g_{\mathrm{s}} \frac{1}{2 \pi} \int d^{2} \widetilde{k} \frac{\left(\frac{1}{2} \widetilde{\varepsilon}_{\mathrm{g}}+\widetilde{k}_{y}^{2}\right)^{2}}{\widetilde{\varepsilon}_{\boldsymbol{k}}^{3}} \delta\left(\widetilde{\omega}-2 \widetilde{\varepsilon}_{\boldsymbol{k}}\right) \\
& =g_{\mathrm{s}} \frac{2}{\pi} \int_{0}^{\infty} \int_{0}^{\infty} d \widetilde{k}_{x} d \widetilde{k}_{y} \frac{\left(\frac{1}{2} \widetilde{\varepsilon}_{\mathrm{g}}+\widetilde{k}_{y}^{2}\right)^{2}}{\left[\sqrt{\left(\frac{1}{2} \widetilde{\varepsilon}_{\mathrm{g}}+\widetilde{k}_{y}^{2}\right)^{2}+\widetilde{k}_{x}^{2}}\right]^{3}} \delta\left(\widetilde{\omega}-2 \sqrt{\left(\frac{1}{2} \widetilde{\varepsilon}_{\mathrm{g}}+\widetilde{k}_{y}^{2}\right)^{2}+\widetilde{k}_{x}^{2}}\right) \\
& =g_{\mathrm{s}} \frac{1}{\pi} \int_{0}^{\infty} \int_{0}^{\infty} d \widetilde{k}_{x} d \widetilde{k}_{y} \frac{\left(\frac{1}{2} \widetilde{\varepsilon}_{\mathrm{g}}+\widetilde{k}_{y}^{2}\right)^{2}}{(\widetilde{\omega} / 2)^{2} \widetilde{k}_{x}} \delta\left(k_{x}-\sqrt{\left(\frac{\widetilde{\omega}}{2}\right)^{2}-\left(\frac{1}{2} \widetilde{\varepsilon}_{\mathrm{g}}+\widetilde{k}_{y}^{2}\right)^{2}}\right) \\
& =g_{\mathrm{s}} \frac{1}{\pi(\widetilde{\omega} / 2)^{2}} \int d \widetilde{k}_{y} \frac{\left(\frac{1}{2} \widetilde{\varepsilon}_{\mathrm{g}}+\widetilde{k}_{y}^{2}\right)^{2}}{\sqrt{\left(\frac{\widetilde{\omega}}{2}\right)^{2}-\left(\frac{1}{2} \widetilde{\varepsilon}_{\mathrm{g}}+\widetilde{k}_{y}^{2}\right)^{2}}}, \\
& \frac{\sigma_{y y}^{\text {inter }}}{\sigma_{0}}=g_{\mathrm{s}} \frac{\pi e^{2}}{\hbar} \int \frac{d^{2} k}{(2 \pi)^{2}} \frac{\left|M_{y}^{+-}(\boldsymbol{k})\right|^{2}}{2 \varepsilon_{+, \boldsymbol{k}}} \delta\left(\hbar \omega-2 \varepsilon_{+, \boldsymbol{k}}\right) \\
& =g_{\mathrm{s}} \frac{2}{\pi} \int d^{2} \widetilde{k}_{\frac{k_{x}}{2}}^{\widetilde{k}_{y}^{2}} \widetilde{\varepsilon}_{\boldsymbol{k}}^{3} \delta\left(\widetilde{\omega}-2 \widetilde{\varepsilon}_{\boldsymbol{k}}\right) \\
& =g_{\mathrm{s}} \frac{8}{\pi} \int_{0}^{\infty} \int_{0}^{\infty} d \widetilde{k}_{x} d \widetilde{k}_{y} \frac{\widetilde{k}_{x}^{2} \widetilde{k}_{y}^{2}}{\left[\sqrt{\left(\frac{1}{2} \widetilde{\varepsilon}_{\mathrm{g}}+\widetilde{k}_{y}^{2}\right)^{2}+\widetilde{k}_{x}^{2}}\right]^{3}} \delta\left(\widetilde{\omega}-2 \sqrt{\left(\frac{1}{2} \widetilde{\varepsilon}_{\mathrm{g}}+\widetilde{k}_{y}^{2}\right)^{2}+\widetilde{k}_{x}^{2}}\right) \\
& =g_{\mathrm{s}} \frac{4}{\pi} \int_{0}^{\infty} \int_{0}^{\infty} d \widetilde{k}_{x} d \widetilde{k}_{y} \frac{\widetilde{k}_{x} \widetilde{k}_{y}^{2}}{(\widetilde{\omega} / 2)^{2}} \delta\left(k_{x}-\sqrt{\left(\frac{\widetilde{\omega}}{2}\right)^{2}-\left(\frac{1}{2} \widetilde{\varepsilon}_{\mathrm{g}}+\widetilde{k}_{y}^{2}\right)^{2}}\right) \\
& =g_{\mathrm{s}} \frac{4}{\pi(\widetilde{\omega} / 2)^{2}} \int d \widetilde{k}_{y} \widetilde{k}_{y}^{2} \sqrt{\left(\frac{\widetilde{\omega}}{2}\right)^{2}-\left(\frac{1}{2} \widetilde{\varepsilon}_{\mathrm{g}}+\widetilde{k}_{y}^{2}\right)^{2}},
\end{aligned}
$$

where $\sigma_{0}=e^{2} /(4 \hbar), \widetilde{\omega}=\hbar \omega / \varepsilon_{0}, \widetilde{\varepsilon}_{\boldsymbol{k}}=\varepsilon_{+}(\boldsymbol{k}) / \varepsilon_{0}=\sqrt{\left(\frac{1}{2} \varepsilon_{g}+\widetilde{k}_{x}^{2}\right)^{2}+\widetilde{k}_{y}^{2}}, f\left(\widetilde{\varepsilon}_{\boldsymbol{k}}\right)=1 /\left[1+e^{\beta\left(\varepsilon_{0} \widetilde{\varepsilon}_{\boldsymbol{k}}-\mu\right)}\right]$ and $g_{\mathrm{s}}=2$ is the spin degeneracy.

Now, consider the undoped case $(\mu=0)$. For the semi-Dirac point $\left(\varepsilon_{\mathrm{g}}=0\right)$, we obtain

$$
\begin{aligned}
\frac{\sigma_{x x}^{\text {inter }}(\omega)}{\sigma_{0}} & =\left[\frac{1}{3 \sqrt{2 \pi}} \frac{\Gamma(1 / 4)}{\Gamma(3 / 4)}\right]\left(\frac{\omega}{\omega_{0}}\right)^{-\frac{1}{2}}, \\
\frac{\sigma_{y y}^{\text {inter }}(\omega)}{\sigma_{0}} & =\left[\frac{8 \sqrt{2}}{5 \sqrt{\pi}} \frac{\Gamma(3 / 4)}{\Gamma(1 / 4)}\right]\left(\frac{\omega}{\omega_{0}}\right)^{\frac{1}{2}} .
\end{aligned}
$$


For the insulator phase $\left(\varepsilon_{\mathrm{g}}>0\right)$, we have

$$
\begin{aligned}
& \frac{\sigma_{x x}^{\text {inter }}(\omega)}{\sigma_{0}}=\frac{2}{3 \pi} \frac{1}{\sqrt{\widetilde{\omega}}}\left[4 p \mathrm{E}\left(\frac{1-p}{2}\right)+(1-2 p) \mathrm{K}\left(\frac{1-p}{2}\right)\right] \Theta\left(\hbar \omega-\varepsilon_{\mathrm{g}}\right), \\
& \frac{\sigma_{y y}^{\text {inter }}(\omega)}{\sigma_{0}}=\frac{8}{15 \pi} \sqrt{\widetilde{\omega}}\left[2\left(3+p^{2}\right) \mathrm{E}\left(\frac{1-p}{2}\right)-(1+p)(3+p) \mathrm{K}\left(\frac{1-p}{2}\right)\right] \Theta\left(\hbar \omega-\varepsilon_{\mathrm{g}}\right),
\end{aligned}
$$

where $p=\varepsilon_{\mathrm{g}} /(\hbar \omega)$. Note that the integral vanishes when $\hbar \omega<\varepsilon_{\mathrm{g}}$, representing an optical gap.

For the Dirac semimetal phase $\left(\varepsilon_{\mathrm{g}}<0\right)$, we find

$$
\begin{aligned}
&\left.\frac{\sigma_{x x}^{\text {inter }}(\omega)}{\sigma_{0}}\right|_{\hbar \omega<\left|\varepsilon_{\mathrm{g}}\right|}=\frac{2 \sqrt{2}}{3 \pi} \frac{1}{\sqrt{\widetilde{\omega}}} \frac{1}{\sqrt{1-p}}\left[2 p(1-p) \mathrm{E}\left(\frac{2}{1-p}\right)+\left(1+2 p^{2}\right) \mathrm{K}\left(\frac{2}{1-p}\right)\right], \\
&\left.\frac{\sigma_{x x}^{\text {inter }}(\omega)}{\sigma_{0}}\right|_{\hbar \omega>\left|\varepsilon_{\mathrm{g}}\right|}=\frac{2}{3 \pi} \frac{1}{\sqrt{\widetilde{\omega}}}\left[4 p \mathrm{E}\left(\frac{1-p}{2}\right)+(1-2 p) \mathrm{K}\left(\frac{1-p}{2}\right)\right] . \\
&\left.\frac{\sigma_{y y}^{\text {inter }}(\omega)}{\sigma_{0}}\right|_{\hbar \omega<\left|\varepsilon_{\mathrm{g}}\right|}=\frac{8 \sqrt{2}}{15 \pi} \sqrt{\widetilde{\omega}} \sqrt{1-p}\left[\left(3+p^{2}\right) \mathrm{E}\left(\frac{2}{1-p}\right)-p(1+p) \mathrm{K}\left(\frac{2}{1-p}\right)\right], \\
&\left.\frac{\sigma_{y y}^{\text {inter }}(\omega)}{\sigma_{0}}\right|_{\hbar \omega>\left|\varepsilon_{\mathrm{g}}\right|}=\frac{8}{15 \pi} \sqrt{\widetilde{\omega}}\left[2\left(3+p^{2}\right) \mathrm{E}\left(\frac{1-p}{2}\right)-(1+p)(3+p) \mathrm{K}\left(\frac{1-p}{2}\right)\right],
\end{aligned}
$$

The effect of non-zero $T$ and $\mu$ on the optical conductivity can be taken into account by multiplying the temperature factor $A(\omega, T, \mu)$ into the $T=0$ and $\mu=0$ case.

\section{Appendix B: Evolution of the optical peaks between the insulator phase and the semi-Dirac point}

In this section, we describe the evolution of the interband optical conductivity along the armchair direction $\left(\sigma_{x x}\right)$ for tetralayer BP from the insulator phase to the semi-Dirac point, as the external electric field increases. Figure 11 shows $\sigma_{x x}$ for tetralayer BP for several values of $E_{\text {ext }}$, and the evolution of the optical peaks as a function of $E_{\text {ext }}$, between the insulator phase and the semi-Dirac point.
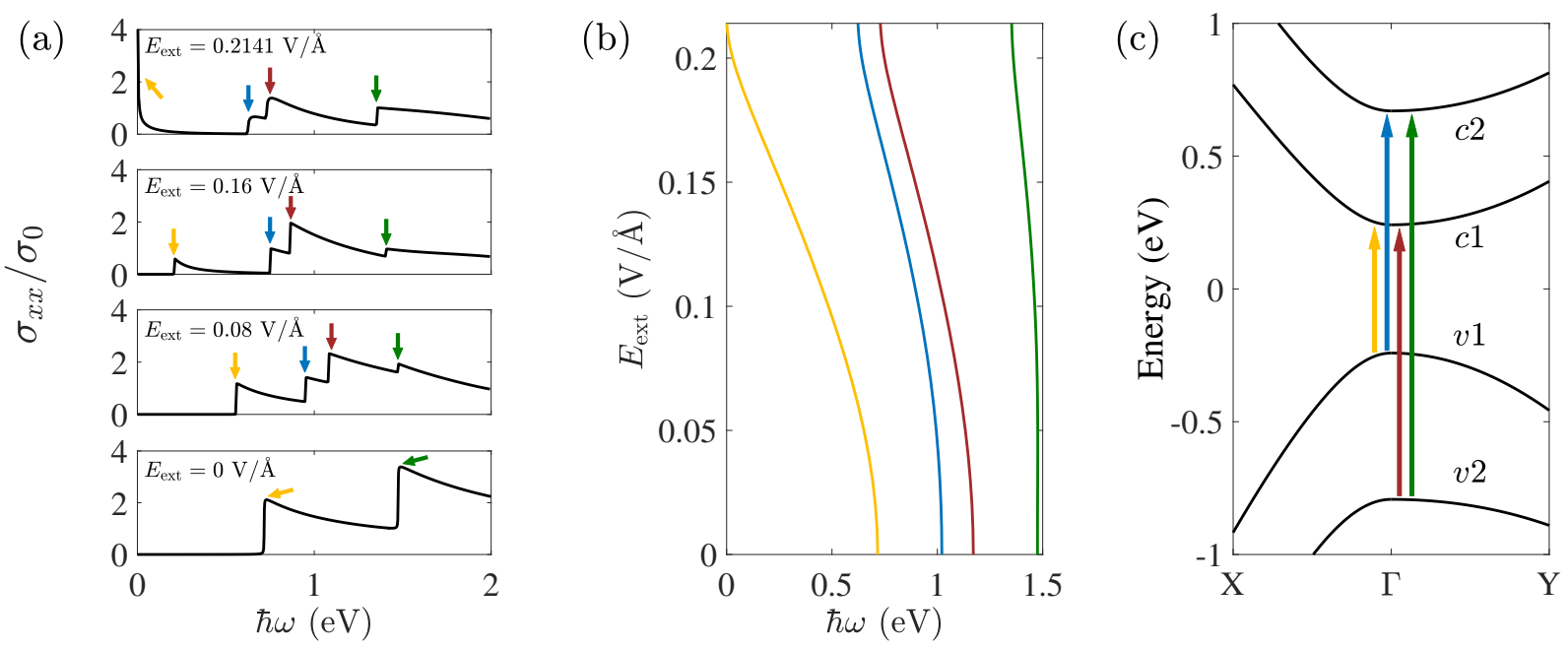

FIG. 11: (a) Optical conductivity $\sigma_{x x}$ of tetralayer BP for $E_{\text {ext }}=0,0.08,0.16,0.2141 \mathrm{~V} / \AA$. (b) Evolution of the first four optical peaks in $\sigma_{x x}$ for tetralayer BP between the insulator phase and the semi-Dirac point. (c) The band structure of tetralayer BP in the insulator phase with $E_{\text {ext }}=0 \mathrm{~V} / \AA$. Arrows indicate the interband transitions corresponding to the first four optical peaks in $\sigma_{x x}$. Here, $\sigma_{0}=\frac{e^{2}}{4 \hbar}$, and the lattice model with $\mu=0$ was utilized for the calculation.

Note that the optical peaks only appear in $\sigma_{x x}$, but they are suppressed in $\sigma_{y y}$ along the zigzag direction, owing to the selection rule. As shown in Fig. 2 in the main text, few-layer BP has reflection symmetry with respect to the $y=0$ plane $\left[\mathcal{M}_{y}:(x, y) \rightarrow(x,-y)\right]$. According to the density functional theory calculation [7, 34, the conduction 
and valence bands at the $\Gamma$ point are composed of just the $3 s, 3 p_{x}$, and $3 p_{z}$ orbitals, which are all even in $\mathcal{M}_{y}$ $\left(\mathcal{M}_{y}\left|3 s, 3 p_{x}, 3 p_{z}\right\rangle=+\left|3 s, 3 p_{x}, 3 p_{z}\right\rangle\right)$. Therefore, the matrix element $M_{y}^{s s^{\prime}}(\boldsymbol{k})$ [Eq. 10 in the main text] at the $\Gamma$ point can be expressed as $M_{y}^{s s^{\prime}}(0)=\left\langle s, 0\left|\frac{\partial H}{\partial k_{y}}\right| s^{\prime}, 0\right\rangle=\left\langle s, 0\left|\mathcal{M}_{y}^{\dagger} \mathcal{M}_{y} \frac{\partial H}{\partial k_{y}} \mathcal{M}_{y}^{\dagger} \mathcal{M}_{y}\right| s^{\prime}, 0\right\rangle=-\left\langle s, 0\left|\frac{\partial H}{\partial k_{y}}\right| s^{\prime}, 0\right\rangle$, leading to $M_{y}^{s s^{\prime}}(0)=0$, and thus suppressing $\sigma_{y y}$.

[1] A. Chaves, W. Ji, J. Maassen, T. Dumitrica and T. Low, 2D Materials: Properties and Devices (2017).

[2] L. Li, J. Kim, C. Jin, G. J. Ye, D. Y. Qiu, H. Felipe, Z. Shi, L. Chen, Z. Zhang, F. Yang et al., Direct observation of the layer-dependent electronic structure in phosphorene, Nat. Nanotechnol. 12, 21 (2017).

[3] J. Qiao, X. Kong, Z.-X. Hu, F. Yang and W. Ji, High-mobility transport anisotropy and linear dichroism in few-layer black phosphorus, Nat. Commun. 5, 4475 (2014).

[4] V. Tran, R. Soklaski, Y. Liang and L. Yang, Layercontrolled band gap and anisotropic excitons in few-layer black phosphorus, Phys. Rev. B 89, 235319 (2014).

[5] R. Fei, V. Tran and L. Yang, Topologically protected Dirac cones in compressed bulk black phosphorus, Phys. Rev. B 91, 195319 (2015).

[6] Z. J. Xiang, G. J. Ye, C. Shang, B. Lei, N. Z. Wang, K. S. Yang, D. Y. Liu, F. B. Meng, X. G. Luo, L. J. Zou, Z. Sun, Y. Zhang and X. H. Chen, Pressure-Induced Electronic Transition in Black Phosphorus, Phys. Rev. Lett. 115, 186403 (2015).

[7] A. Rodin, A. Carvalho and A. C. Neto, Strain-induced gap modification in black phosphorus, Phys. Rev. Lett. 112, 176801 (2014).

[8] Q. Liu, X. Zhang, L. B. Abdalla, A. Fazzio and A. Zunger, Switching a normal insulator into a topological insulator via electric field with application to phosphorene, Nano Lett. 15, 1222 (2015).

[9] S. Yuan, E. van Veen, M. I. Katsnelson and R. Roldán, Quantum Hall effect and semiconductor-to-semimetal transition in biased black phosphorus, Phys. Rev. B 93, 245433 (2016).

[10] H. Doh and H. J. Choi, Dirac-semimetal phase diagram of two-dimensional black phosphorus, 2D Mater. 4, 025071 (2017).

[11] J. Kim, S. S. Baik, S. H. Ryu, Y. Sohn, S. Park, B.-G. Park, J. Denlinger, Y. Yi, H. J. Choi and K. S. Kim, Observation of tunable band gap and anisotropic Dirac semimetal state in black phosphorus, Science 349, 723 (2015).

[12] S. S. Baik, K. S. Kim, Y. Yi and H. J. Choi, Emergence of Two-Dimensional Massless Dirac Fermions, Chiral Pseudospins, and Berrys Phase in Potassium Doped FewLayer Black Phosphorus, Nano Lett. 15, 7788 (2015).

[13] J. Kim, S. S. Baik, S. W. Jung, Y. Sohn, S. H. Ryu, H. J. Choi, B.-J. Yang and K. S. Kim, Two-Dimensional Dirac Fermions Protected by Space-Time Inversion Symmetry in Black Phosphorus, Phys. Rev. Lett. 119, 226801 (2017).

[14] L. Li, Y. Yu, G. J. Ye, Q. Ge, X. Ou, H. Wu, D. Feng, X. H. Chen and Y. Zhang, Black phosphorus field-effect transistors, Nat. Nanotechnol. 9, 372 (2014).
[15] P. Li and I. Appelbaum, Electrons and holes in phosphorene, Phys. Rev. B 90, 115439 (2014).

[16] C. Han, M. Yao, X. Bai, L. Miao, F. Zhu, D. Guan, S. Wang, C. Gao, C. Liu, D. Qian et al., Electronic structure of black phosphorus studied by angle-resolved photoemission spectroscopy, Phys. Rev. B 90, 085101 (2014).

[17] T. Low, A. S. Rodin, A. Carvalho, Y. Jiang, H. Wang, F. Xia and A. H. Castro Neto, Tunable optical properties of multilayer black phosphorus thin films, Phys. Rev. B 90, 075434 (2014).

[18] F. Xia, H. Wang and Y. Jia, Rediscovering black phosphorus as an anisotropic layered material for optoelectronics and electronics, Nat. Commun. 5, 4458 (2014).

[19] S. Yuan, A. Rudenko and M. Katsnelson, Transport and optical properties of single-and bilayer black phosphorus with defects, Phys. Rev. B 91, 115436 (2015).

[20] N. Mao, J. Tang, L. Xie, J. Wu, B. Han, J. Lin, S. Deng, W. Ji, H. Xu, K. Liu et al., Optical anisotropy of black phosphorus in the visible regime, J. Am. Chem. Soc. 138, 300 (2015).

[21] H. Yuan, X. Liu, F. Afshinmanesh, W. Li, G. Xu, J. Sun, B. Lian, A. G. Curto, G. Ye, Y. Hikita et al., Polarization-sensitive broadband photodetector using a black phosphorus vertical $\mathrm{p}-\mathrm{n}$ junction, Nat. Nanotechnol. 10, 707 (2015).

[22] C. Lin, R. Grassi, T. Low and A. S. Helmy, Multilayer black phosphorus as a versatile mid-infrared electro-optic material, Nano Lett. 16, 1683 (2016).

[23] W. S. Whitney, M. C. Sherrott, D. Jariwala, W.-H. Lin, H. A. Bechtel, G. R. Rossman and H. A. Atwater, Field Effect Optoelectronic Modulation of Quantum-Confined Carriers in Black Phosphorus, Nano Lett. 17, 78 (2017).

[24] R. Peng, K. Khaliji, N. Youngblood, R. Grassi, T. Low and M. Li, Midinfrared Electro-optic Modulation in FewLayer Black Phosphorus, Nano Lett. 17, 6315 (2017).

[25] Z. Torbatian and R. Asgari, Optical absorption properties of few-layer phosphorene, Phys. Rev. B 98, 205407 (2018).

[26] R. A. Doganov, E. C. OFarrell, S. P. Koenig, Y. Yeo, A. Ziletti, A. Carvalho, D. K. Campbell, D. F. Coker, K. Watanabe, T. Taniguchi et al., Transport properties of pristine few-layer black phosphorus by van der Waals passivation in an inert atmosphere, Nat. Commun. 6, 6647 (2015).

[27] P. Adroguer, D. Carpentier, G. Montambaux and E. Orignac, Diffusion of Dirac fermions across a topological merging transition in two dimensions, Phys. Rev. B 93, 125113 (2016).

[28] Y. Liu and P. P. Ruden, Temperature-dependent anisotropic charge-carrier mobility limited by ionized impurity scattering in thin-layer black phosphorus, Phys. Rev. B 95, 165446 (2017). 
[29] S. Park, S. Woo and H. Min, Semiclassical Boltzmann transport theory of few-layer black phosphorus in various phases, 2D Materials 6, 025016 (2019).

[30] J. M. Pereira and M. I. Katsnelson, Landau levels of single-layer and bilayer phosphorene, Phys. Rev. B 92, 075437 (2015).

[31] M. Tahir, P. Vasilopoulos and F. Peeters, Magnetooptical transport properties of monolayer phosphorene, Phys. Rev. B 92, 045420 (2015).

[32] X. Zhou, W.-K. Lou, F. Zhai and K. Chang, Anomalous magneto-optical response of black phosphorus thin films, Phys. Rev. B 92, 165405 (2015).

[33] X. Y. Zhou, R. Zhang, J. P. Sun, Y. L. Zou, D. Zhang, W. K. Lou, F. Cheng, G. H. Zhou, F. Zhai and K. Chang, Landau levels and magneto-transport property of monolayer phosphorene, Sci. Rep. 5, 12295 (2015).

[34] A. N. Rudenko and M. I. Katsnelson, Quasiparticle band structure and tight-binding model for single-and bilayer black phosphorus, Phys. Rev. B 89, 201408 (2014).

[35] A. N. Rudenko, S. Yuan and M. I. Katsnelson, Toward a realistic description of multilayer black phosphorus: From $G W$ approximation to large-scale tightbinding simulations, Phys. Rev. B 92, 085419 (2015).

[36] D. J. P. de Sousa, L. V. de Castro, D. R. da Costa, J. M. Pereira and T. Low, Multilayered black phosphorus: From a tight-binding to a continuum description, Phys. Rev. B 96, 155427 (2017).

[37] D. Çakır, C. Sevik and F. M. Peeters, Significant effect of stacking on the electronic and optical properties of fewlayer black phosphorus, Phys. Rev. B 92, 165406 (2015).
[38] R. J. Wu, M. Topsakal, T. Low, M. C. Robbins, N. Haratipour, J. S. Jeong, R. M. Wentzcovitch, S. J. Koester and K. A. Mkhoyan, Atomic and electronic structure of exfoliated black phosphorus, Journal of Vacuum Science \& Technology A: Vacuum, Surfaces, and Films 33, 060604 (2015).

[39] S. Banerjee and W. E. Pickett, Phenomenology of a semiDirac semi-Weyl semimetal, Phys. Rev. B 86, 075124 (2012).

[40] P. V. Sriluckshmy, K. Saha and R. Moessner, Interplay between topology and disorder in a two-dimensional semi-Dirac material, Phys. Rev. B 97, 024204 (2018).

[41] H. Min, B. Sahu, S. K. Banerjee and A. MacDonald, Ab initio theory of gate induced gaps in graphene bilayers, Phys. Rev. B 75, 155115 (2007).

[42] L. Li, B. Partoens and F. Peeters, Tuning the electronic properties of gated multilayer phosphorene: A selfconsistent tight-binding study, Phys. Rev. B 97, 155424 (2018).

[43] G. D. Mahan, Many-particle physics, Springer Science \& Business Media (2013).

[44] G. Y. Cho and E.-G. Moon, Novel quantum criticality in two dimensional topological phase transitions, Sci. Rep. 6, 19198 (2016).

[45] H. Isobe, B.-J. Yang, A. Chubukov, J. Schmalian and N. Nagaosa, Emergent Non-Fermi-Liquid at the Quantum Critical Point of a Topological Phase Transition in Two Dimensions, Phys. Rev. Lett. 116, 076803 (2016). 